\title{
STRONG MEASURE ZERO SETS WITHOUT COHEN REALS
}

January 1991

\section{Martin Goldstern ${ }^{1}$}

\author{
Bar Ilan University \\ Haim Judah ${ }^{1}$ \\ Bar Ilan University and U.C. Chile \\ Saharon Shelah ${ }^{1,2}$ \\ Hebrew University of Jerusalem
}

\begin{abstract}
If ZFC is consistent, then each of the following are consistent with ZFC $+2^{\aleph_{0}}=\aleph_{2}$ :

1. $X \subseteq \mathbb{R}$ is of strong measure zero iff $|X| \leq \aleph_{1}+$ there is a generalized Sierpinski set.

2. The union of $\aleph_{1}$ many strong measure zero sets is a strong measure zero set + there is a strong measure zero set of size $\aleph_{2}$.
\end{abstract}

1 The authors thank the Israel Foundation for Basic Research, Israel Academy of Science.

${ }^{2}$ Publication 438 


\section{$\S$ 0. Introduction}

In this paper we continue the study of the structure of strong measure zero sets. Strong measure zero sets have been studied from the beginning of this century. They were discovered by E. Borel, and Luzin, Sierpinski, Rothberger and others turned their attention to the structure of these sets and proved very interesting mathematical theorems about them. Most of the constructions of strong measure zero sets involve Luzin sets, which have a strong connection with Cohen reals (see [6]). In this paper we will show that this connection is only apparent; namely, we will build models where there are strong measure zero sets of size c without adding Cohen reals over the ground model.

Throughout this work we will investigate questions about strong measure zero sets under the assumption that $\mathbf{c}=2^{\aleph_{0}}=\aleph_{2}$. The reason is that $\mathrm{CH}$ makes many of the questions we investigate trivial, and there is no good technology available to deal with most of our problems when $2^{\aleph_{0}}>\aleph_{2}$.

0.1 Definition: A set $X \subseteq \mathbb{R}$ of reals has strong measure zero if for every sequence $\left\langle\varepsilon_{i}: i<\omega\right\rangle$ of positive real numbers there is a sequence $\left\langle x_{i}: i<\omega\right\rangle$ of real numbers such that

$$
X \subseteq \bigcup_{i<\omega}\left(x_{i}-\varepsilon_{i}, x_{i}+\varepsilon_{i}\right)
$$

We let $\mathcal{S} \subset \mathbf{P}(\mathbb{R})$ be the ideal of strong measure zero sets.

0.2 Remark: (a) if we work in ${ }^{\omega_{2}}$ then $X \subseteq{ }^{\omega_{2}}$ has strong measure zero if

$$
\left(\forall h \in \omega^{\omega} \omega\right)\left(\exists g \in \prod_{n}^{h(n)} 2\right)(\forall x \in X)\left(\exists^{\infty} n\right)(g(n)=x \mid h(n))
$$

or equivalently,

$$
\left(\forall h \in \omega_{\omega}\right)\left(\exists g \in \prod_{n}^{h(n)} 2\right)(\forall x \in X)(\exists n)(g(n)=x \mid h(n))
$$

(b) To every question about strong measure zero sets in $\mathbb{R}$ there is a corresponding question about a strong measure zero set of $\omega_{2}$, and for all the questions we consider the corresponding answers are the same. So we will work sometimes in $\mathbb{R}$, sometimes in $\omega_{2}$.

0.3 Definition: Assume that $\mathcal{H} \subseteq \omega_{\omega}$. We say that $\bar{\nu}$ has index $\mathcal{H}$, if $\bar{\nu}=\left\langle\nu^{h}: h \in \mathcal{H}\right\rangle$ and for all $h \in \mathcal{H}, \nu^{h}$ is a function with domain $\omega$ and $\forall n \nu^{h}(n) \in{ }^{h(n)_{2}}$. We let

$$
X_{\bar{\nu}}:=\bigcap_{h \in \mathcal{H}} \bigcup_{k \in \omega}\left[\nu^{h}(k)\right]
$$

(where we let $[\eta]:=\left\{f \in \omega^{\omega}: \eta \subseteq f\right\}$ ).

We say that $X_{\bar{\nu}}$ is the set "defined" by $\bar{\nu}$. 
0.4 Fact: Assume $\mathcal{H} \subseteq \omega_{\omega}$ is a dominating family, i.e., for all $f \in \omega_{\omega}$ there is $h \in \mathcal{H}$ such that $\forall n f(n)<h(n)$. Then:

(1) If $\bar{\nu}$ has index $\mathcal{H}$, then $X_{\bar{\nu}}$ is a strong measure zero set.

(2) If $X$ is a strong measure zero set, then there is a sequence $\bar{\nu}$ with index $\mathcal{H}$ such that $X \subseteq X_{\bar{\nu}}$.

0.5 Definition: A set of reals $X \subseteq \mathbb{R}$ is a GLuzin (generalized Luzin) set if for every meager set $M \subseteq \mathbb{R}, X \cap M$ has cardinality less than c. $X$ is a generalized Sierpinski set if set if for every set $M \subseteq \mathbb{R}$ of Lebesgue measure $0, X \cap M$ has cardinality less than c.

0.6 Fact: (a) If c is regular, and $X$ is GLuzin, then $X$ has strong measure zero.

(b) A set of mutually independent Cohen reals over a model $M$ is a GLuzin set.

(c) If $\mathbf{c}>\aleph_{1}$ is regular, and $X$ is a GLuzin set, then $X$ contains Cohen reals over $L$.

Proof: See [6].

0.7 Theorem: [6] Con $(\mathrm{ZF})$ implies Con $(\mathrm{ZFC}+$ there is a GLuzin set which is not strong measure zero).

0.8 Theorem: $[6] \operatorname{Con}(\mathrm{ZF})$ implies $\operatorname{Con}\left(\mathrm{ZFC}+\mathbf{c}>\aleph_{1}+\exists X \in[\mathbb{R}]^{\mathbf{c}}, X\right.$ a strong measure zero set + there are no GLuzin sets).

In theorem 0.16 we will show a stronger form of 0.8 .

\subsection{Definition:}

(1) Let Unif $(\mathcal{S})$ be the following statement: "Every set of reals of size less than $\mathbf{c}$ is a strong measure zero set."

(2) We say that the ideal of strong measure zero sets is c-additive, or $\operatorname{Add}(\mathcal{S})$, if for every $\kappa<\mathbf{c}$ the union of $\kappa$ many strong measure zero sets is a strong measure zero set. (So $\operatorname{Add}(\mathcal{S}) \Rightarrow \operatorname{Unif}(\mathcal{S})$.)

0.10 Remark: Rothberger ([13] and [12]) proved that the following are equivalent:

(i) $\operatorname{Unif}(\mathcal{S})$

(ii) for every $h: \omega \rightarrow \omega$, for every $F \in\left[\prod_{n} h(n)\right]^{<\mathbf{c}}$, there exists $f^{*} \in \omega_{\omega}$ such that for every $f \in F$ there are infinitely many $n$ satisfying $f(n)=f^{*}(n)$.

Miller ([10]) noted that this implies the following:

$$
\operatorname{Add}(\mathcal{M}) \quad \text { iff } \quad \operatorname{Unif}(\mathcal{S}) \text { and } \mathbf{b}=\mathbf{c}
$$

(See 0.17 for definitions)

Rothberger proved interesting results about the existence of strong measure zero sets, namely:

If $\mathbf{b}=\aleph_{1}$, then there is a strong measure zero set of size $\aleph_{1}$. (See [5].)

In this spirit, we first prove the following result: 
0.11 Theorem: If $\operatorname{Unif}(\mathcal{S})$ and $\mathbf{d}=\mathbf{c}$, then there exists a strong measure zero set of size c.

We start the proof by proving the following

0.12 Fact: If $\mathbf{d}=\mathbf{c}$, then there is a set $\left\{f_{i}: i<\mathbf{c}\right\}$ of functions in ${ }^{\omega} \omega$ such that for every $g \in{ }^{\omega} \omega$, the set

$$
\left\{i<\mathbf{c}: f_{i} \leq^{*} g\right\}
$$

has cardinality less than $\mathbf{c}$.

Proof of the fact: We build $\left\langle f_{i}: i<\mathbf{c}\right\rangle$ by transfinite induction. Let $\omega_{\omega}=\left\{g_{j}: j<\mathbf{c}\right\}$. We will ensure that for $j<i, f_{i} \nless^{*} g_{j}$. This will be sufficient.

But this is easy to achieve, as for any $i$, the family $\left\{g_{j}: j<i\right\}$ is not dominating, so there exists a function $f_{i}$ such that for all $j<i$, for infinitely many $n, f_{i}(n)>g_{j}(n)$.

This completes the proof of 0.12 .

0.13 Proof of 0.11: Using $\mathbf{d}=\mathbf{c}$, let $\left\langle f_{i}: i<\mathbf{c}\right\rangle$ be a sequence as in 0.12. Let $F:{ }^{\omega} \omega \rightarrow$ $[0,1]-\mathbf{Q}$ be a homeomorphism. ( $\mathbf{Q}$ is the set of rational numbers.) We will show that $X:=\left\{F\left(f_{i}\right): i<\mathbf{c}\right\}$ is a strong measure zero set.

Let $\left\langle\varepsilon_{n}: n<\omega\right\rangle$ be a sequence of positive numbers. Let $\left\{r_{n}: n \in \omega\right\}=\mathbf{Q}$. Then $U_{1}:=\bigcup_{n \in \omega}\left(r_{n}-\varepsilon_{2 n}, r_{n}+\varepsilon_{2 n}\right)$ is an open set. So $K:=[0,1]-U_{1}$ is closed, hence compact. As $K \subseteq \operatorname{rng}(F)$, also $F^{-1}(K) \subseteq{ }^{\omega} \omega$ is a compact set. So for all $n$ the projection of $F^{-1}(K)$ to the $n$th coordinate is a compact (hence bounded) subset of $\omega$, say $\subseteq g(n)$. So

$$
F^{-1} K \subseteq\left\{f \in{ }^{\omega} \omega: f \leq^{*} g\right\}
$$

Let $Y:=X-U_{1} \subseteq K$. Then $Y \subseteq F\left(F^{-1}(K)\right) \subseteq\left\{F\left(f_{i}\right): f_{i} \leq^{*} g\right\}$ is (by assumption on $\left.\left\langle f_{i}: i<\mathbf{c}\right\rangle\right)$ a set of size $<\mathbf{c}$, hence has strong measure zero. So there exists a sequence of real numbers $\left\langle x_{n}: n<\omega\right\rangle$ such that $Y \subseteq U_{2}$, where

$$
U_{2}:=\bigcup_{n \in \omega}\left(x_{n}-\varepsilon_{2 n+1}, x_{n}+\varepsilon_{2 n+1}\right)
$$

and $X \subseteq U_{1} \cup U_{2}$. So $X$ is indeed a strong measure zero set.

In section 2 we will build models where $\operatorname{Add}(\mathcal{S})$ holds and the continuum is bigger than $\aleph_{1}$ without adding Cohen reals. First we will show in 3.4:

0.14 Theorem: If ZFC is consistent, then

$$
\mathrm{ZFC}+\mathbf{c}=\aleph_{2}+\mathcal{S}=[\mathbb{R}]^{\leq \aleph_{1}}+\text { there are no Cohen reals over } L
$$
is consistent.

Note that $\mathbf{c}=\aleph_{2}$ and $\mathcal{S}=[\mathbb{R}]^{\leq \aleph_{1}}$ implies

(1) $\operatorname{Add}(\mathcal{S})$. (Trivially)

(2) $\mathbf{b}=\mathbf{d}=\aleph_{1}$. (By 0.11) 
The same result was previously obtained by Corazza[3]. In his model the nonexistence of strong measure zero sets of size $\mathbf{c}$ is shown by proving that every set of size $\mathbf{c}$ can be mapped uniformly continuously onto the unit interval (which is impossible for a strong measure zero set). Thus, the question arises whether is possible to get a model of $\mathcal{S}=[\mathbb{R}]^{<\mathbf{c}}+$ $\mathbf{c}=\aleph_{2}+$ "not all set of size $\mathbf{c}$ can be continuously mapped onto $[0,1] . "$

By adding random reals to our construction, we answer this question in the following stronger theorem:

0.15 Theorem: If ZFC is consistent, then

$$
\begin{gathered}
\mathrm{ZFC}+\mathbf{c}=\aleph_{2}+\mathcal{S}=[\mathbb{R}] \leq \aleph_{1}+\text { there are no Cohen reals over } L \\
+ \text { there is a generalized Sierpinski set }
\end{gathered}
$$

is consistent. (See 0.5.)

By a remark of Miller $[8, \S 2]$ a generalized Sierpinski set cannot be mapped continuously onto $[0,1]$ (not even with a Borel function).

Pawlikowski [11] showed that the additivity of the ideal $\mathcal{S}$ of strong measure zero sets does not imply the additivity of the ideal $\mathcal{M}$ of meager sets. For this he built a model satisfying $\operatorname{Add}(\mathcal{S})+\mathbf{c}=\aleph_{2}+\mathbf{b}=\aleph_{1}$. He used a finite support iteration of length $\omega_{2}$. So he adds many Cohen reals, and in the final model $\operatorname{Cov}(\mathcal{M})$ holds (i.e., $\mathbb{R}$ can not be written as the union of less than $\mathbf{c}$ many meager sets). We will improve his result in the next theorem:

0.16 Theorem: If ZFC is consistent, then

$$
\mathrm{ZFC}+\mathbf{c}=\mathbf{d}=\aleph_{2}>\mathbf{b}+\mathbf{A d d}(\mathcal{S})+\text { no real is Cohen over } L
$$

is consistent.

(Note that by $0.11, \mathbf{d}=\mathbf{c}+\operatorname{Add}(\mathcal{S})$ implies that there is a strong measure zero set of size c.)

0.17 Notation: We use standard set-theoretical notation. We identify natural numbers $n$ with their set of predecessors, $n=\{0, \ldots, n-1\}$. ${ }^{A} B$ is the set of functions from $A$ into $B, A^{<\omega}:=\bigcup_{n<\omega}{ }^{n} A .|A|$ denotes the cardinality of a set $A . \mathbf{P}(A)$ is the power set of a set $A, A \subset B$ means $A \subseteq B \& A \neq B . A-B$ is the set-theoretic difference of $A$ and $B$. $[A]^{\kappa}:=\{X \subseteq A:|X|=\kappa\} .[A]^{<\kappa}$ and $[A]^{\leq \kappa}$ are defined similarly. (We write $A:=B$ or $B=: A$ to mean: the expression $B$ defines the term (or constant) $A$.)

Ord is the set of ordinals. $c f(\alpha)$ is the cofinality of an ordinal $\alpha . S_{\beta}^{\alpha}:=\left\{\delta \in \omega_{\beta}: c f(\delta)=\right.$ $\left.\omega_{\alpha}\right\}$. In particular, $S_{2}^{1}$ is the set of all ordinals $<\omega_{2}$ of uncountable cofinality.

$\mathbb{R}$ is the set of real numbers. $\mathbf{c}=|\mathbb{R}|$ is the size of the continuum. For $f, g \in \omega_{\omega} \omega$ we let $f<g$ iff for all $n f(n)<g(n)$, and $f<^{*} g$ if for some $n_{0} \in \omega, \forall n \geq n_{0} f(n)<g(n)$. The "bounding number" $\mathbf{b}$ and the "dominating number" $\mathbf{d}$ are defined as

$$
\begin{aligned}
\mathbf{b} & :=\min \left\{|\mathcal{H}|: \mathcal{H} \subseteq{ }^{\omega} \omega, \forall g \in{ }^{\omega}{ }_{\omega} \exists h \in \mathcal{H} \neg\left(h<^{*} g\right)\right\} \\
\mathbf{d} & :=\min \left\{|\mathcal{H}|: \mathcal{H} \subseteq{ }^{\omega} \omega, \forall g \in{ }^{\omega}{ }_{\omega} \exists h \in \mathcal{H} g<h\right\} \\
& =\min \left\{|\mathcal{H}|: \mathcal{H} \subseteq{ }^{\omega}{ }_{\omega}, \forall g \in{ }^{\omega_{\omega}} \exists h \in \mathcal{H} g<^{*} h\right\}
\end{aligned}
$$

(It is easy to see $\omega_{1} \leq \mathbf{b} \leq \mathbf{d} \leq \mathbf{c}$.) 
We call a set $\mathcal{H} \subseteq{ }^{\omega} \omega$ dominating, if $\forall g \in{ }^{\omega} \omega \exists h \in \mathcal{H} g<h$.

$\mathcal{M}$ is the ideal of meager subsets of $\mathbb{R}$ (or of $\omega_{2}$ ). $\mathcal{S}$ is the ideal of strong measure zero sets. For any ideal $\mathcal{J} \subset \mathbf{P}(\mathbb{R}), \operatorname{Add}(\mathcal{J})$ abbreviates the statement: "The union of less than $\mathbf{c}$ many sets in $\mathcal{J}$ is in $\mathcal{J}$." $\operatorname{Cov}(\mathcal{J})$ means that the reals cannot be covered by less than $\mathbf{c}$ many sets in $\mathcal{J}$.

If $f$ is a function, $\operatorname{dom}(f)$ is the domain of $f$, and $\operatorname{rng}(f)$ is the range of $f$. For $A \subseteq \operatorname{dom}(f)$, $f \mid A$ is the restriction of $f$ to $A$. For $\eta \in 2^{<\omega},[\eta]:=\left\{f \in{ }^{\omega} 2: \eta \subseteq f\right\}$.

0.18 More Notation: If $Q$ is a forcing notion, $G_{Q}$ is the canonical name for the generic filter on $Q$. We interpret $p \leq q$ as $q$ is stronger (or "has more information") than $p$. (So $\left.p \leq q \Rightarrow q \mid \vdash p \in G_{Q}.\right)$

When we deal with a (countable support) iteration $\left\langle P_{\alpha}, Q_{\alpha}: \alpha<\varepsilon\right\rangle$, we write $G_{\alpha}$ for the canonical name of the generic filter on $\alpha$, and $G(\alpha)$ for the generic filter on $Q_{\alpha}$. If there is a natural way to associate a "generic" real to the generic filter on $Q_{\alpha}$, we write $g_{\alpha}$ for the real given by $G(\alpha)$. We write $\mid \vdash_{\alpha}$ for the forcing relation of $P_{\alpha}$. If $\beta<\alpha, G_{\beta}$ always stands for $G_{\alpha} \cap P_{\beta} . V=V_{0}$ is the ground model, and $V_{\alpha}=V\left[G_{\alpha}\right]$. $P_{\varepsilon}$ is the countable support limit of $\left\langle P_{\alpha}: \alpha<\varepsilon\right\rangle$. $P_{\varepsilon} / G_{\alpha}$ is the $P_{\alpha}$-name for $\left\{p \in P_{\varepsilon}: p \mid \alpha \in G_{\alpha}\right\}$ (with the same $\leq$ relation as $P_{\varepsilon}$ ). The forcing relation with respect to $P_{\varepsilon} / G_{\alpha}$ (in $V_{\alpha}$ ) is denoted by $\vdash \vdash_{\alpha \varepsilon}$.

There is a natural dense embedding from $P_{\varepsilon}$ into $P_{\alpha} * P_{\varepsilon} / G_{\alpha}$. Thus we always identify $P_{\alpha}$-names for $P_{\varepsilon} / G_{\alpha}$-names with the corresponding $P_{\varepsilon}$-names.

$\emptyset_{\alpha}$ is the weakest condition of $P_{\alpha}$, and $\emptyset_{\alpha} \mid \vdash_{\alpha} \varphi$ is usually abbreviated to $\mid \vdash_{\alpha} \varphi$. $\mid \vdash_{\alpha}\left(\mid \vdash_{\alpha \delta} \varphi\right)$ iff $\left.\mid \vdash_{\delta} \varphi\right)$.

0.19 Even more Notation: The following notation is used when we deal with trees of finite sequences:

For $\eta \in V^{<\omega}, i \in V, \eta \frown i$ is the function $\eta \cup\{\langle|\eta|, i\rangle\} \in V^{<\omega}$. $p \subseteq \omega^{<\omega}$ is a tree if $p \neq \emptyset$, and for all $\eta \in p$, all $k<|\eta|, \eta \mid k \in p$. Elements of a tree are often called "nodes". We call $|\eta|$ the "length" of $\eta$. We reserve the word "height" for the notion defined in $\mathbf{2 . 2}$.

For $p \subseteq \omega^{<\omega}, \eta \in p$, we let $\operatorname{succ}_{p}(\eta):=\{i: \eta \frown i \in p\}$.

If $p$ is a tree, $\eta \in p$, let $p^{[\eta]}:=\{\nu \in p: \eta \subseteq \nu$ or $\nu \subseteq \eta\}$.

If $p \subseteq \omega^{<\omega}$ is a tree, $b \subseteq p$ is called a branch, if $b$ is a maximal subset of $p$ that is linearly ordered by $\subseteq$.

Clearly, if $\forall \eta \in p \operatorname{succ}_{p}(\eta) \neq \emptyset$, then a subset $b \subseteq p$ is a branch iff $b$ is of the form $b=\{f \mid n: n \in \omega\}$ for some $f \in \omega_{\omega}^{\omega}$.

We let $\operatorname{stem}(p)$ be the intersection of all branches of $p$. 


\section{A few well known facts}

We collect a few more or less well known facts about forcing, for later reference.

1.1 Definition: An ultrafilter $\mathcal{U}$ on $\omega$ is called a P-Point, if for any sequence $\left\langle A_{n}: n \in \omega\right\rangle$ of sets in $\mathcal{U}$ there is a set $A$ in $\mathcal{U}$ that is almost contained in every $A_{n}$ (i.e., $\forall n A-A_{n}$ is finite).

1.2 Definition: For any ultrafilter $\mathcal{U}$ on $\omega$, we define the P-point game $G(\mathcal{U})$ as follows: There are two players, "IN" and "NOTIN". The game consists of $\omega$ many moves.

In the $n$-th move, player NOTIN picks a set $A_{n} \in \mathcal{U}$, and player IN picks a finite set $a_{n} \subseteq A_{n}$.

Player IN wins if after $\omega$ many moves, $\bigcup_{n} a_{n} \in \mathcal{U}$.

We write a play (or run) of $G(\mathcal{U})$ as

$$
\left\langle A_{0} ; a_{0} \rightarrow A_{1} ; a_{1} \rightarrow A_{2} ; \ldots\right\rangle
$$

It is well known that an ultrafilter $\mathcal{U}$ is a P-point iff player NOTIN does not have a winning strategy in the P-point game.

For the sake of completeness, we give a proof of the nontrivial implication " $\Rightarrow$ " (which is all we will need later):

Let $\mathcal{U}$ be a P-point, and let $\sigma$ be a strategy for player NOTIN. We will construct a run of the game in which player NOTIN followed $\sigma$, but IN won.

Let $A_{0}$ be the first move according to $\sigma$. For each $n$, let $\mathcal{A}_{n}$ be the set of all responses of player notin according to $\sigma$ in an initial segment of a play of length $\leq n$ in which player IN has played only subsets of $n$ :

$$
\begin{aligned}
\mathcal{A}_{n}:=\left\{A_{k}:\right. & k \leq n,\left\langle A_{0} ; a_{0} \rightarrow A_{1} ; \ldots ; a_{k-1} \rightarrow A_{k}\right\rangle \text { is an } \\
& \text { initial segment of a play in which NOTIN } \\
& \text { obeyed } \left.\sigma, \text { and } a_{0}, \ldots, a_{k-1} \subseteq n\right\}
\end{aligned}
$$

Note that $\mathcal{A}_{0}=\left\{A_{0}\right\}$, and for all $n, \mathcal{A}_{n}$ is a finite subset of $\mathcal{U}$.

As $\mathcal{U}$ is a $\mathrm{P}$-point, there is a set $X \in \mathcal{U}$ such that for all $A \in \bigcup_{n} \mathcal{A}_{n}, X-A$ is finite.

Let $X \subseteq A_{0} \cup n_{0}$, and for $k>0$ let $n_{k}$ satisfy

$$
n_{k}>n_{k-1} \quad \text { and } \quad \forall A \in \mathcal{A}_{n_{k-1}} X \subseteq A \cup n_{k}
$$

Either $\bigcup_{k \in \omega}\left[n_{2 k}, n_{2 k+1}\right) \in \mathcal{U}$, or $\bigcup_{k \in \omega}\left[n_{2 k+1}, n_{2 k+2}\right) \in \mathcal{U}$.

Without loss of generality we assume $\bigcup_{k \in \omega}\left[n_{2 k}, n_{2 k+1}\right) \in \mathcal{U}$.

Now define a play $\left\langle A_{0} ; a_{0} \rightarrow A_{1} ; a_{1} \rightarrow A_{2} ; \ldots\right\rangle$ of the game $G(\mathcal{U})$ by induction as follows:

$A_{0}$ is given.

Given $A_{j}$, let $a_{j}:=A_{j} \cap\left[n_{2 j}, n_{2 j+1}\right)$ and let $A_{j+1}$ be $\sigma$ 's response to $a_{j}$. 
Then as $a_{0}, \ldots, a_{j-1} \subseteq n_{2 j}$, we have $X \subseteq A_{j} \cup n_{2 j}$ for all $j$. Therefore for all $j$ we have $X \cap\left[n_{2 j}, n_{2 j+1}\right) \subseteq\left(A_{j} \cup n_{2 j}\right) \cap\left[n_{2 j}, n_{2 j+1}\right)=A_{j} \cap\left[n_{2 j}, n_{2 j+1}\right)=a_{j}$. So $\bigcup_{j \in \omega} a_{j} \supseteq$ $X \cap \bigcup_{j \in \omega}\left[n_{2 j}, n_{2 j+1}\right) \in \mathcal{U}$.

Thus player IN wins the play $\left\langle A_{0} ; a_{0} \rightarrow A_{1} ; a_{1} \rightarrow A_{2} ; \ldots\right\rangle$ in which player NOTIN obeyed $\sigma$.

1.3 Definition: We say that a forcing notion $Q$ preserves P-points, if for every P-point ultrafilter $\mathcal{U}$ on $\omega, \vdash_{Q}$ "U⿱UU generates an ultrafilter", i.e. $\mid \vdash_{Q} \forall x \in \mathbf{P}(\omega) \exists u \in \mathcal{U}(u \subseteq$ $x$ or $u \subseteq \omega-x)$."

[9] defined the following forcing notion:

1.4 Definition: "Rational perfect set forcing", $R P$ is defined as the set of trees $p \subseteq \omega^{<\omega}$ satisfying

(1) for all $\eta \in p,\left|\operatorname{succ}_{p}(\eta)\right| \in\left\{1, \aleph_{0}\right\}$ (See 0.19)

(2) for all $\eta \in p$ there is $\nu \in p$ with $\eta \subseteq \nu$ and $\left|\operatorname{succ}_{p}(\eta)\right|=\aleph_{0}$.

We let $p \geq q$ iff $p \subseteq q$.

Then the following hold:

\subsection{Lemma:}

(1) $R P$ preserves P-points. $([9,4.1])$

(2) $R P$ adds an unbounded function. $([9, \S 2])$

(3) $R P$ is proper. (This is implicit in [9]. See also 2.16)

The next lemma can be found, e.g., in [7, VII ?? and Exercise H2]:

1.6 Fact: If $Q$ is a forcing notion satisfying the $\aleph_{2}$-cc, then

(1) If $\mid \vdash_{Q} \stackrel{c}{\sim}: \omega_{2}^{V} \rightarrow \omega_{2}^{V}$, then there is a function $c: \omega_{2} \rightarrow \omega_{2}$ such that

$\vdash \vdash_{Q} \forall \alpha<\omega_{2}: \underset{\sim}{c}(\alpha)<c(\alpha)$.

(2) $\vdash \vdash_{Q} \aleph_{2}^{V}=\aleph_{2}$

(3) For every stationary $S \subseteq \aleph_{2}, \vdash_{Q}$ "S $S$ is stationary on $\aleph_{2}$ ".

The following fact is from $[14, \mathrm{~V} 4.4]$ :

1.7 Fact: Assume $\left\langle P_{\alpha}, Q_{\alpha}: \alpha<\omega_{2}\right\rangle$ is an iteration of proper forcing notions $Q_{\alpha}$. Then for every $\delta \leq \omega_{2}$ of cofinality $>\omega, \vdash_{\delta} \omega_{\omega} \cap V_{\delta}={ }^{\omega} \omega \cap \bigcup_{\alpha<\delta} V_{\alpha}$, or in other words: "no new reals appear in limit stages of cofinality $>\omega$ ".

As a consequence, $\mid \vdash_{\omega_{2}}$ "If $X \subseteq \omega_{\omega} \omega,|X| \leq \aleph_{1}$, then there is $\delta<\omega_{2}$ such that $X \in V_{\delta}$."

We also recall the following facts about iteration of proper forcing notions:

1.8 Lemma: Assume $C H$, and let $\left\langle P_{\alpha}, Q_{\alpha}: \alpha<\omega_{2}\right\rangle$ be a countable support iteration such that for all $\alpha<\omega_{2}, \mid \vdash_{\alpha}$ "Q $Q_{\alpha}$ is a proper forcing notion of size $\leq \mathbf{c}$."

Then

(1) $\forall \alpha<\omega_{2}: \vdash_{\alpha} \mathbf{c}=\aleph_{1}$. (see [14, III 4.1]) 
(2) $\mid \vdash_{\omega_{2}} \mathbf{c} \leq \aleph_{2}$. (This follows from 1.7 and (1))

(3) For all $\alpha \leq \omega_{2}, P_{\alpha}$ is proper [14, III 3.2] and satisfies the $\aleph_{2}$-cc. (See [14, III 4.1])

(4) $\mid \vdash_{\omega_{2}} \aleph_{1}^{V}=\aleph_{1}$. (See $[14$, III 1.6])

In $[2,4.1]$ the following is proved:

1.9 Lemma: Assume $\left\langle P_{\alpha}, Q_{\alpha}: \alpha<\omega_{2}\right\rangle$ is as in 1.8, and for all $\alpha<\omega_{2}$ :

$$
\vdash \vdash_{\alpha} \text { "Q } Q_{\alpha} \text { preserves P-points." }
$$

Then for all $\alpha \leq \omega_{2}, P_{\alpha}$ preserves P-points.

1.10 Definition: We say that a forcing notion $Q$ is $\omega_{\omega}$-bounding, if the set of "old" functions is a dominating family in the generic extension by $Q$, or equivalently,

$$
\mid \vdash_{Q} \forall f \in \omega_{\omega} \exists g \in \omega_{\omega} \cap V \forall n f(n)<g(n)
$$

[14, V 4.3] proves:

1.11 Lemma: Assume $\left\langle P_{\alpha}, Q_{\alpha}: \alpha<\omega_{2}\right\rangle$ is as in 1.8, and for all $\alpha<\omega_{2}$ :

$$
\vdash_{\alpha} \text { " } Q_{\alpha} \text { is } \omega_{\omega} \text {-bounding and } \omega \text {-proper." }
$$

Then for all $\alpha \leq \omega_{2}, P_{\alpha}$ is $\omega_{\omega}$-bounding.

(We may even replace $\omega$-proper by "proper", see [14], [4])

The following is trivial to check:

1.12 Fact: Assume $Q$ is a forcing notion that preserves $\mathrm{P}$-points or is ${ }_{\omega} \omega$-bounding. Then

$$
\vdash_{Q} \text { "There are no Cohen reals over } V \text { " }
$$

1.13 Definition: A forcing notion $P$ is strongly $\omega_{\omega}$-bounding, if there is a sequence $\left\langle\leq_{n}: n \in \omega\right\rangle$ of binary reflexive relations on $P$ such that for all $n \in \omega$ :

(1) $p \leq_{n} q \Rightarrow p \leq q$.

(2) $p \leq_{n+1} q \Rightarrow p \leq_{n} q$.

(3) If $p_{0} \leq_{0} p_{1} \leq_{1} p_{2} \leq_{3} \cdots$, then there is a $q$ such that $\forall n p_{n+1} \leq_{n} q$.

(4) If $p \mid \vdash$ " $\alpha$ is an ordinal," and $n \in \omega$, then there exists $q \geq_{n} p$ and a finite set $A \subseteq$ Ord such that $Q \mid \vdash \alpha \in A$.

1.14 Definition: (1) If $\left\langle P_{\alpha}, Q_{\alpha}: \alpha<\varepsilon\right\rangle$ is an iteration of strongly $\omega_{\omega}$-bounding forcing notions, $F \subseteq \varepsilon$ finite, $n \in \omega, p, q \in P_{\varepsilon}$, we say that $p \leq_{F, n} q$ iff $p \leq q$ and $\forall \alpha \in$ $F q|\alpha| \vdash p(\alpha) \leq_{n} q(\alpha)$.

(2) A sequence $\left\langle\left\langle p_{n}, F_{n}\right\rangle: n \in \omega\right\rangle$ is called a fusion sequence if $\left\langle F_{n}: n \in \omega\right\rangle$ is an increasing family of finite subsets of $\varepsilon,\left\langle p_{n}: n \in \omega\right\rangle$ is an increasing family of conditions in $P_{\varepsilon}$, $\forall n p_{n} \leq_{n, F_{n}} p_{n+1}$ and $\bigcup_{n} \operatorname{dom}\left(p_{n}\right) \subseteq \bigcup_{n} F_{n}$.

Note that 1.13 is not a literally a strengthening of Baumgarter's "Axiom A" (see [1]), as we do not require that the relations $\leq_{n}$ are transitive, and in (2) we only require $p_{n+1} \leq_{n} q$ rather than $p_{n+1} \leq_{n+1} q$. Nevertheless, the same proof as in [1] shows the following fact: 


\subsection{Fact:}

(1) If the sequence $\left\langle\left\langle p_{n}, F_{n}\right\rangle: n \in \omega\right\rangle$ is a fusion sequence, then there exists a condition $q \in P_{\varepsilon}$ such that for all $n \in \omega, p_{n+1} \geq_{F_{n}, n} q$.

(2) If $\underset{\sim}{\alpha}$ is a $P_{\varepsilon}$-name of an ordinal, $n \in \omega, F \subseteq P_{\varepsilon}$ finite, then for all $p$ there exists a condition $q \geq_{n, F} p$ and a finite set $A$ of ordinals such that $q \mid \vdash \underset{\sim}{\sim} \in A$.

(3) If $\underset{\sim}{X}$ is a $P_{\varepsilon}$-name of a countable set of ordinals, $n \in \omega, F \subseteq P_{\varepsilon}$ finite, then for all $p$ there exists a condition $q \geq_{n, F} p$ and a countable set $A$ of ordinals such that $q \mid \vdash \underset{\sim}{X} \subseteq A$.

The next fact is also well known:

1.16 Fact: Let $B$ be the random real forcing. Then $B$ is strongly $\omega_{\omega}$-bounding.

[Proof: Conditions in $B$ are Borel subsets of $[0,1]$ of positive measure, $p \leq q$ iff $p \supseteq q$. We let $p \leq_{n} q$ iff $p \leq q$ and $\mu(p-q) \leq 10^{-n-1} \mu(p)$, where $\mu$ is the Lebesgue measure. Then if $p_{0} \geq_{0} p_{1} \geq_{1} \cdots$, letting $q:=\bigcap_{n} p_{n}$ we have for all $n$, all $k \geq n, \mu\left(p_{k}-p_{k+1}\right) \leq$ $10^{-k-1} \mu\left(p_{k}\right) \leq 10^{-k-1} \mu\left(p_{n}\right)$, so $\mu\left(p_{n}-q\right) \leq 10^{-n-1}+10^{-n-2}+\cdots \leq 2 * 10^{-n-1} \mu\left(p_{n}\right)$. In particular, $\mu(q) \geq 0.8 * \mu\left(p_{0}\right)$, so $q$ is a condition, and $q \geq_{n-1} p_{n}$ for all $n>0$.

Given a name $\underset{\sim}{\alpha}$, an integer $n$ and a condition $p$ such that $p \mid \vdash^{-}$" $\alpha$ is an ordinal," let $A$ be the set of all ordinals $\beta$ such that $\llbracket \underset{\sim}{\alpha}=\beta \rrbracket \cap p$ has positive measure $(\llbracket \varphi \rrbracket$ is the boolean value of the statement $\varphi$, i.e. the union of all conditions forcing $\varphi)$. Since $\sum_{\beta \in A} \mu(\llbracket \underset{\sim}{\alpha}=$ $\beta \rrbracket \cap p)=\mu(p)$ there is a finite subset $F \subseteq A$ such that letting $q:=p \cap \bigcup_{\beta \in A \llbracket \alpha=\beta \rrbracket \text { we }}$ have $\mu(q) \geq\left(1-10^{-n-1}\right) \mu(p)$. So $q \geq_{n} p$ and $q \mid \vdash \alpha \in F$.]

We will also need the following lemma from [17, §5, Theorem 9]:

1.17 Lemma: Every stationary $S \subseteq \aleph_{2}$ can be written as a union of $\aleph_{2}$ many disjoint stationary sets.

Finally, we will need the following easy fact (which is true for any forcing notion $Q$ )

1.18 Fact: If $f$ is a $Q$-name for a function from $\omega$ to $\omega, \vdash \vdash_{Q} f \notin V$, and $r_{0}, r_{1}$ are any two conditions in $\widetilde{Q}$, then there are $l \in \omega, j_{0} \neq j_{1}, r_{0}^{\prime} \geq r_{0}, r_{1}^{\prime} \geq r_{1}$ such that $r_{0}^{\prime} \mid \vdash f(l)=j_{0}$, $r_{1}^{\prime} \mid \vdash f(l)=j_{1}$.

[Proof: There are a function $f_{0}$ and a sequence $r_{0}=r^{0} \leq r^{1} \leq \cdots$ of conditions in $Q$ such that for all $n, r^{n}|\vdash f| n=f_{0} \mid n$. Since $r_{1}\left|\vdash f \notin V, r_{1}\right| \vdash \exists l \underset{\sim}{f}(l) \neq f_{0}(l)$. There is a condition $r_{1}^{\prime} \geq r_{1}$ such that for some $l \in \omega$ and some $j_{1} \neq f_{0}(l), r_{1}^{\prime} \widetilde{\uparrow} f(l)=j_{1}$. Let $j_{0}:=f_{0}(l)$, and let $r_{0}^{\prime}:=r^{l+1}$.] 


\section{$\S 2$ H-perfect trees}

In this section we describe a forcing notion $P T_{H}$ that we will use in an iteration in the next section. We will prove the following properties of $P T_{H}$ :

(a) $P T_{H}$ is proper and $\omega_{\omega}$-bounding.

(b) $P T_{H}$ preserves P-points.

(c) $P T_{H}$ does not "increase" strong measure zero sets defined in the ground model.

(d) $P T_{H}$ makes the reals of the ground model (and hence, by (c), the union of all strong measure zero sets defined in the ground model) a strong measure zero set.

2.1 Definition: For each function $H$ with domain $\omega$ satisfying $\forall n \in \omega 1<|H(n)|<\omega$, we define the forcing $P T_{H}$, the set of $H$-perfect trees to be the set of all $p$ satisfying

(A) $p \subseteq \omega^{<\omega}$ is a tree.

(B) $\forall \eta \in p \forall l \in \operatorname{dom}(\eta): \eta(l) \in H(l)$.

(C) $\forall \eta \in p:\left|\operatorname{succ}_{p}(\eta)\right| \in\{1,|H(|\eta|)|\}$.

(D) $\forall \eta \in p \exists \nu \in p: \eta \subseteq \nu,\left|\operatorname{succ}_{p}(\nu)\right|=|H(|\nu|)|$.

\subsection{Definition:}

(1) For $p \in P T_{H}$, we let the set of "splitting nodes" of $p$ be

$$
\operatorname{split}(p):=\left\{\eta \in p:\left|\operatorname{succ}_{p}(\eta)\right|>1\right\}
$$

(2) The height of a node $\eta \in p \in P T_{H}$ is the number of splitting nodes strictly below $\eta$ :

$$
\operatorname{ht}_{p}(\eta):=|\{\nu \subset \eta: \nu \in \operatorname{split}(p)\}|
$$

(Note that $\operatorname{ht}_{p}(\eta) \leq|\eta|$. )

(3) For $p \in P T_{H}, k \in \omega$, we let the $k$ th splitting level of $p$ be the set of splitting nodes of height $k$.

$$
\operatorname{split}_{k}(p):=\left\{\eta \in \operatorname{split}(p): \operatorname{ht}_{p}(\eta)=k\right\}
$$

$\left(\right.$ Note that $\left.\operatorname{split}_{0}(p)=\{\operatorname{stem}(p)\}.\right)$

(4) For $u \subseteq \omega$, we let

$$
\operatorname{split}^{u}(p):=\bigcup_{k \in u} \operatorname{split}_{k}(p)
$$

\subsection{Remarks:}

(i) Since $H(n)$ is finite, (3) just means that either $\eta$ has a unique successor $\eta \frown i$, or $\operatorname{succ}_{p}(\eta)=H(|\eta|)$.)

(ii) Letting $H^{\prime}(n)=|H(n)|$, clearly $P T_{H}$ is isomorphic to $P T_{H^{\prime}}$ (and the obvious isomorphism respects the functions $\eta \mapsto \operatorname{ht}_{p}(\eta),\langle p, k\rangle \mapsto \operatorname{split}_{k}(p)$, etc) 
2.4 Remark: If we let $H(n)=\omega$ for all $n$, then 2.1(A)-(D) define $R P$, rational perfect set forcing. The definitions in 2.2 make sense also for this forcing. Since we will not use the fact that $H(n)$ is finite before $2.12,2.5-2.11$ will be true also for $R P$.

2.5 Fact: Let $p, q \in P T_{H}, n \in \omega, \eta, \nu \in \omega^{<\omega}$. Then

(1) If $\eta \subset \nu \in p$, then $\operatorname{ht}_{p}(\eta) \leq \operatorname{ht}_{p}(\nu)$. If moreover $\eta \in \operatorname{split}(p)$, then $\operatorname{ht}_{p}(\eta)<\operatorname{ht}_{p}(\nu)$.

(2) If $b \subseteq p$ is a branch, then $b \cap \operatorname{split}_{n}(p) \neq \emptyset$.

(3) If $p \supseteq q$, then for all $n, q \cap \operatorname{split}_{n}(p) \neq \emptyset$.

(4) If $\eta \in p$ and $\operatorname{ht}_{p}(\eta) \leq n$ then $\exists \nu \in p, \eta \subseteq \nu$ and $\nu \in \operatorname{split}_{n}(p)$.

(5) If $\eta_{0} \neq \eta_{1}$ are elements of $\operatorname{split}_{n}(p)$, then $\eta_{0} \nsubseteq \eta_{1}$, and $\eta_{1} \nsubseteq \eta_{0}$.

Proof: (1) is immediate form the definition of ht.

For (2), it is enough to see that $b \cap \operatorname{split}(p)$ is infinite. (Then ordering $b$ by inclusion, the $n$th element of $b \cap \operatorname{split}(p)$ will be in $\operatorname{split}_{n-1}(p)$.)

So assume that $b \cap \operatorname{split}(p)$ is finite. Recall that each $\eta \in b-\operatorname{split}(p)$ has a unique successor in $p$. By $2.1(\mathrm{C}), b$ cannot have a last element, so $b$ is infinite. Hence there is $\eta_{0} \in b$ such that

$$
\forall \nu \in b: \eta_{0} \subseteq \nu \Rightarrow\left|\operatorname{succ}_{p}(\nu)\right|=1 .
$$

A trivial induction on $|\nu|$ shows that this implies

$$
\forall \nu \in p: \eta_{0} \subseteq \nu \Rightarrow \nu \in b
$$

Hence

$$
\forall \nu \in p: \eta_{0} \subseteq \nu \Rightarrow\left|\operatorname{succ}_{p}(\nu)\right|=1
$$

This contradicts 2.1(D).

To prove (3), let $b$ be any branch of $q$. $b$ is also a branch of $p$, so (2) shows that $q \cap \operatorname{split}_{n}(p) \supseteq$ $b \cap \operatorname{split}_{n}(p) \neq \emptyset$.

Proof of (4): Let $b$ be a branch of $p$ containing $\eta$. By (2) there is $\nu \in b \cap \operatorname{split}_{n}(p)$. If $\nu \subset \eta$, then $\mathrm{ht}_{p}(\eta)>\mathrm{ht}_{p}(\nu)=n$, which is impossible. Hence $\eta \subseteq \nu$.

(5) follows easily from (1).

2.6 Definition: For $p, q \in P T_{H}, n \in \omega$, we let

(1) $p \leq q$ (" $q$ is stronger than $p$ ") iff $q \subseteq p$.

(2) $p \leq_{n} q$ iff $p \leq q$ and $\operatorname{split}_{n}(p) \subseteq q$. (So also $\operatorname{split}_{k}(p) \subseteq q$ for all $k<n$.)

2.7 Fact: If $p \leq_{n} q, n>0$, then $\operatorname{stem}(p)=\operatorname{stem}(q)$.

2.8 Fact: Assume $p, q \in P T_{H}, n \in \omega, p \leq_{n} q$.

(0) For all $\eta \in q$, ht $_{q}(\eta) \leq \operatorname{ht}_{p}(\eta)$.

(1) For all $k \leq n$, $\operatorname{split}_{k}(p) \subseteq \operatorname{split}(q)$.

(2) For all $k<n, \operatorname{split}_{k}(p)=\operatorname{split}_{k}(q)$. 
(3) If $p \leq_{n} q \leq_{n} r$, then $p \leq_{n} r$.

Proof: (0) is clear.

(1): Let $\eta \in \operatorname{split}_{k}(p)$ for some $k<n$, then by 2.5(4) there is a $\nu, \eta \subseteq \nu \in \operatorname{split}_{n}(p) \subseteq q$, so $\eta \in q$.

(2): Let $\eta \in \operatorname{split}_{k}(p)$, then $\eta \in \operatorname{split}(q)$. Clearly $\operatorname{ht}_{q}(\eta) \leq \mathrm{ht}_{p}(\eta)=k$. Using (1) inductively, we also get $\operatorname{ht}_{q}(\eta) \geq k$.

(3): Let $\eta \in \operatorname{split}_{n}(p)$. So $\eta \in q, \operatorname{ht}_{q}(\eta) \leq \mathrm{ht}_{p}(\eta)=n$. By 2.5(4), there is $\nu \in \operatorname{split}_{n}(q)$, $\eta \subseteq \nu$. As $\nu \in r, \eta \in r$.

2.9 Definition and Fact: If $p_{0} \leq_{1} p_{1} \leq_{2} p_{2} \leq_{3} \cdots$ are conditions in $P T_{H}$, then we call the sequence $\left\langle p_{n}: n<\omega\right\rangle$ a "fusion sequence". If $\left\langle p_{n}: n<\omega\right\rangle$ is a fusion sequence, then

(1) $p_{\infty}:=\bigcap_{n \in \omega} p_{n}$ is in $P T_{H}$

(2) For all $n: p_{n} \leq_{n+1} p_{\infty}$.

\subsection{Fact:}

(1) If $\eta \in p \in P T_{H}$, then $p^{[\eta]} \in P T_{H}$, and $p \leq p^{[\eta]}$. (See 0.19.)

(2) If $p \leq q$ are conditions in $P T_{H}, \eta \in q$, then $p^{[\eta]} \leq q^{[\eta]}$.

2.11 Fact: If for all $\eta \in \operatorname{split}_{n}(p), q_{\eta} \geq p^{[\eta]}$ is a condition in $P T_{H}$, then

(1) $q:=\bigcup_{\eta \in \text { split }_{n}(p)} q_{\eta}$ is in $P T_{H}$,

(2) $q \geq_{n} p$

(3) for all $\eta \in \operatorname{split}_{n}(p), q^{[\eta]}=q_{\eta}$.

2.12 Fact: If $n \in \omega, p \in P T_{H}$, then $\operatorname{split}_{n}(p)$ is finite.

Proof: This is the first time that we use the fact that each $H(n)$ is a finite set: Assume that the conclusion is not true, so for some $n$ and $p, \operatorname{split}_{n}(p)$ is infinite. Then also

$$
T:=\left\{\eta\left|k: \eta \in \operatorname{split}_{n}(p), k \leq\right| \eta \mid\right\} \subseteq p
$$

is infinite. As $T$ is a finitely splitting tree, there has to be an infinite branch $b \subseteq T$. By $2.5(2)$, there is $\nu \in b \subseteq T, \mathrm{ht}_{p}(\nu)>n$. This is a contradiction to $2.5(1)$.

2.13 Fact: $P T_{H}$ is strongly $\omega_{\omega}$-bounding, i.e.:

If $\underset{\sim}{\alpha}$ is a $P T_{H}$-name for an ordinal, $p \in P T_{H}, n \in \omega$, then there exists a finite set $A$ of ordinals and a condition $q \in P T_{H}, p \leq_{n} q$, and $q \mid \vdash \alpha \in A$.

Proof: Let $C:=\operatorname{split}_{n}(p)$. $C$ is finite. For each node $\eta \in C$, let $q_{\eta} \geq p^{[\eta]}$ be a condition such that for some ordinal $\alpha_{\eta} q_{\eta} \mid \vdash \underset{\sim}{\sim}=\alpha_{\eta}$. Now let

$$
q:=\bigcup_{\eta \in C} q_{\eta} \quad \text { and } \quad A:=\left\{\alpha_{\eta}: \eta \in C\right\}
$$

Since any extension of $q$ must be compatible with some $q^{[\eta]}$ (for some $\left.\eta \in C\right), q \mid \vdash \alpha \in A$. 
2.14 Corollary: $P T_{H}$ is proper (and indeed satisfies axiom A, so is $\alpha$-proper for any $\left.\alpha<\omega_{1}\right)$ and ${ }^{\omega} \omega$-bounding. Moreover, if $n \in \omega, p \in P T_{H}, \tau$ a name for a set of ordinals, then there exists a condition $q \geq_{n} p$ such that

(1) If $p \mid \vdash$ " $\tau$ is finite", then there is a finite set $A$ such that $q \mid \vdash$ " $\tau \subseteq A$ ".

(2) If $p \mid \vdash$ " $\tau$ is countable", then there is a countable set $A$ such that $q \mid \vdash$ " $\tau \subseteq A$ ".

Proof: Use 2.13 and 2.9 .

Similarly to 2.13 we can show:

2.15 Fact: Assume that $\underset{\sim}{\alpha}$ is a $R P$-name for an ordinal, $p \in R P, n \in \omega$.

Then there exists a countable set $A$ of ordinals and a condition $q \in P T_{H}, p \leq_{n} q$, and $q \mid \vdash \alpha \in A$.

Proof: Same as the proof of 2.13, except that now the set $C$ and hence also the set $A$ may be countable.

2.16 Fact: $R P$ is proper (and satifies axiom A). Proof: By 2.15 and 2.9.

2.17 Definition: Let $G \subseteq P T_{H}$ be a $V$-generic filter. Then we let $\underset{\sim}{g}$ be the $P T_{H}$-name defined by

$$
\underset{\sim}{g}:=\bigcap_{p \in G} p
$$

We may write $\underset{\sim}{g} H$ or $\underset{\sim}{g} P T_{H}$ for this name $\underset{\sim}{g}$. If $P T_{H}$ is the $\alpha$ th iterand $Q_{\alpha}$ in an iteration, we write $\underset{\sim}{g}$ for $\underset{\sim}{g}{\underset{\sim}{H}}_{H}$

2.18 Fact: $\emptyset_{P T_{H}}$ forces that

(0) $g$ is a function with domain $\omega$,

(1) $\widetilde{\forall} \underset{\sim}{g}(n) \in H(n)$.

(2) For all $f \in V$, if $\forall n f(n) \in H(n)$ then $\exists^{\infty} n f(n)=\underset{\sim}{g}(n)$.

Furthermore, for all $p \in P T_{H}$,

(3) $p \mid \vdash$ " $\{\underset{\sim}{g} \mid n: n \in \omega\}$ is a branch through $p$.

(4) $p|\vdash \forall k \underset{\sim}{\widetilde{\sim} g}| n \in \operatorname{split}_{k}(p)$

Proof: (0) and (2) are straightforward density arguments. (1) and (3) follow immedaitely from the definition of $\underset{\sim}{g}$. (4) follows from (3) and 2.5(2), applied in $V^{P T_{H}}$.

2.19 Remark: Since $\operatorname{Unif}(\mathcal{S})$ is equivalent to for every $H: \omega \rightarrow \omega$, for every $F \in\left[\prod_{n} H(n)\right]^{<\mathbf{c}}$, there exists $f^{*} \in \omega_{\omega} \omega$ such that for every $f \in F$ there are infinitely many $n$ satisfying $f(n)=f^{*}(n)$,

2.18(2) shows that if we have $\mathbf{c}=\aleph_{2}$ and Martin's Axiom for the forcing notions $P T_{H}$ (for all $H$ ), then we also have $\operatorname{Unif}(\mathcal{S})$. (In fact the "easy" implication " $\Leftarrow$ " of this equivalence is sufficient.) This can be achieved by a countable support iteration of length $\aleph_{2}$ of forcing notions $P T_{H}$, with the usual bookkeeping argument (as in [16]). 
We will show a stronger result in 3.3: If $P:=P_{\omega_{2}}$ is the limit of a countable support iteration $\left\langle P_{\alpha}, Q_{\alpha}: \alpha<\omega_{2}\right\rangle$, where "many" $Q_{\alpha}$ are of the form $P T_{H_{\alpha}}$ for some $H_{\alpha}$, then some bookkeeping argument can ensure that $V^{P} \models \operatorname{Add}(\mathcal{S})$.

Since $P T_{H}$ is ${ }^{\omega} \omega$-bounding, it does not add Cohen reals. The same is true for a countable support iteration of forcings of the form $P T_{H}$. However, in 3.9 we will have to consider a forcing iteration in which some forcing notions are of the form $P T_{H}$, but others do add an unbounded real. To establish that even these iterations do not add Cohen reals, we will need the fact that the forcing notion $P T_{H}$ preserves many ultrafilters.

2.20 Definition: Let $Q$ be a forcing notion, $\underset{\sim}{x}$ a $Q$-name, $p \in Q, p \mid \vdash \underset{\sim}{\sim} \subseteq \omega$. We say that $x^{*} \subseteq \omega$ is an interpretation of $\underset{\sim}{x}$ (above $p$ ), if for all $n$ there is a condition $p_{n} \geq p$ such that $p_{n} \mid \vdash x \cap n=x^{*} \cap n$.

2.21 Fact: Assume $Q, p, \underset{\sim}{\sim}$ are as in 2.20. Then

(1) There exists $x^{*} \subseteq \omega$ such that $x^{*}$ is an interpretation of $\underset{\sim}{x}$ above $p$.

(2) If $p \leq p^{\prime}$ and $x^{*}$ is an interpretation of $\underset{\sim}{x}$ above $p^{\prime}$, then $x^{*}$ is an interpretation of $\underset{\sim}{x}$ above $p$.

2.22 Lemma: $P T_{H}$ preserves $\mathrm{P}$-points, i.e.: If $\mathcal{U} \in V$ is a $\mathrm{P}$-point ultrafilter on $\omega$, then

$$
\mid \vdash_{P T_{H}} \text { "U⿱U generates an ultrafilter." }
$$

Proof: Assume that the conclusion is false. Then there is a $P T_{H}$-name $\mathcal{\tau}$ for a subset of $\omega$ and a condition $p_{0}$ such that

$$
p_{0}\left|\vdash_{P T_{H}} \forall x \in \mathcal{U}:\right| x \cap \underset{\sim}{\sim}|=|(\omega-x) \cap \underset{\sim}{\sim} \mid=\aleph_{0}
$$

For each $p \in P T_{H}$ we choose a set $\tau(p)$ such that

- $\tau(p)$ is an interpretation of $\underset{\tau}{\sim}$ above $p$.

- If there is an interpretation of $\mathcal{\tau}$ above $p$ that is an element of $\mathcal{U}$, then $\tau(p) \in \mathcal{U}$.

Note that if $\tau(p) \in \mathcal{U}$ and $p \geq p^{\prime}$, then also $\tau\left(p^{\prime}\right) \in \mathcal{U}$, since (by 2.21(2)) we could have chosen $\tau\left(p^{\prime}\right):=\tau(p)$. Hence either for all $p \tau(p) \in \mathcal{U}$, or for some $p_{1} \geq p_{0}$, all $p \geq p_{1}$, $\tau(p) \notin \mathcal{U}$. In the second case we let $\underset{\sim}{\sim}$ be a name for the complement of $\underset{\sim}{\sim}$, and let $\sigma(p)=\omega-\tau(p)$. Then $\sigma(p) \in \mathcal{U}$ for all $p \geq p_{1}$. Also, $\sigma(p)$ is an interpretation of $\sigma$ above $p$.

So wlog for all $p \geq p_{1}, \tau(p) \in \mathcal{U}$ for some $p_{1} \in P T_{H}, p_{1} \geq p_{0}$.

We will show that there is a condition $q \geq p_{1}$ and a set $a \in \mathcal{U}$ such that $q \mid \vdash a \subseteq \mathcal{\tau}$.

Recall that as $\mathcal{U}$ is a $\mathrm{P}$-point, player NOTIN does not have a winning strategy in the P-point game for $\mathcal{U}$ (see 1.2).

We now define a strategy for player NOTIN. On the side, player NOTIN will construct a fusion sequence $\left\langle p_{n}: n<\omega\right\rangle$ and a sequence $\left\langle m_{n}: n<\omega\right\rangle$ of natural numbers. 
$p_{0}$ is given.

Given $p_{n}$, we let

$$
A_{n}=\bigcap_{\eta \in \operatorname{split}_{n+1}\left(p_{n}\right)} \tau\left(p_{n}{ }^{[\eta]}\right)
$$

This set is in $\mathcal{U}$. Player IN responds with a finite set $a_{n} \subseteq A_{n}$. Let $m_{n}:=1+\max \left(a_{n}\right)$. For each $\eta \in \operatorname{split}_{n+1}\left(p_{n}\right)$ there is a condition $q_{\eta} \geq p_{n}{ }^{[\eta]}$ forcing $\underset{\sim}{\sim} \cap m_{n}=\tau\left(p_{n}{ }^{[\eta]}\right) \cap m_{n}$, so in particular

$$
q_{\eta} \mid \vdash a_{n} \subseteq \tau \cap m_{n}
$$

Let $p_{n+1}=\bigcup_{\eta \in \operatorname{split}_{n+1}\left(p_{n}\right)} q_{\eta}$.

Then

$$
p_{n+1} \geq_{n+1} p_{n} \quad \text { and } \quad p_{n+1} \mid \vdash a_{n} \subseteq \underset{\sim}{\sim}
$$

This is a well-defined strategy for player NOTIN. As it is not a winning strategy, there is a play in which IN wins. During this play, we have constructed a fusion sequence $\left\langle p_{n}: n\langle\omega\rangle\right.$. Letting $a:=\bigcup_{n} a_{n}, q:=\bigcap_{n} p_{n}$, we have that $a \in \mathcal{U}, p_{0} \leq q \in P T_{H}$ (by 2.9), and $q \mid \vdash a \subseteq \underset{\sim}{\sim}($ by $(*))$, a contradiction to our assumption.

The following facts will be needed for the proof that if we iterate forcing notions $P T_{H}$ with carefully chosen functions $H$, then we will get a model where the ideal of strong measure zero sets is c-additive.

2.23 Fact and Definition: Assume $p \in P T_{H}, u \subseteq \omega$ is infinite, $v=\omega-u$. Then we can define a stronger condition $q$ by "trimming" $p$ at each node in $\operatorname{split}^{v}(p)$. (See 2.2(4).) Formally, let $\vec{\imath}=\left\langle i_{\eta}: \eta \in \operatorname{split}^{v}(p)\right\rangle$ be a sequence satisfying $i_{\eta} \in H(|\eta|)$ for all $\eta \in \operatorname{split}^{v}(p)$.

Then

$$
p_{\vec{\imath}}:=\left\{\eta \in p: \forall n \in \operatorname{dom}(\eta): \text { If } \eta \mid n \in \operatorname{split}^{v}(p), \text { then } \eta(n)=i_{\eta \mid n}\right\}
$$

is a condition in $P T_{H}$

Proof: Let $q:=p_{\vec{\imath}} . q$ satisfies $(\mathrm{A})-(\mathrm{B})$ of the definition 2.1 of $P T_{H}$. The definition of $p_{\vec{\imath}}$ immediately implies:

(1) If $\eta \in \operatorname{split}^{v}(p) \cap q$, then $\operatorname{succ}_{q}(\eta)=\left\{i_{\eta}\right\}$.

(2) If $\eta \in \operatorname{split}^{u}(p) \cap q$, then $\operatorname{succ}_{q}(\eta)=\operatorname{succ}_{p}(\eta)=H(|\eta|)$.

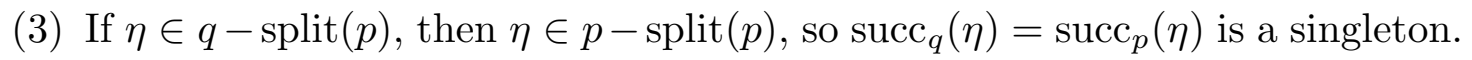

Note that $\operatorname{split}(p)=\operatorname{split}^{u}(p) \cup \operatorname{split}^{v}(p)$, so (1)-(3) cover all possible cases for $\eta \in q$.

So $q$ also satisfies $2.1(\mathrm{C})$.

From (1)-(3) we can also conclude:

(4) For all $\eta \in q$ : $\operatorname{succ}_{q}(\eta) \neq \emptyset$. 
To show that $q \in P T_{H}$, we still have to check condition 2.1(D). So let $\eta \in q$. Since $u$ is infinite, there is $k \in u, k>|\eta|$. By (4), there is an infinite branch $b \subseteq q$ containing $\eta$. By 2.5(2) there is $\nu \in b, \operatorname{ht}_{p}(\nu)=k$. Then $\eta \subseteq \nu$, and $\nu \in \operatorname{split}(q)$.

2.24 Fact: $p_{\vec{\imath}} \mid \vdash^{*} \eta \subseteq \underset{\sim}{g} \& \eta \in \operatorname{split}^{v}(p) \Rightarrow \underset{\sim}{g}(|\eta|)=i_{\eta}$ " (where $\underset{\sim}{g}$ is a name for the generic branch defined in 2.18).

Proof: $p_{\vec{\imath}} \mid \vdash g \subseteq p_{\vec{\imath}}$ and $\operatorname{succ}_{p_{\vec{\imath}}}(\eta)=\left\{i_{\eta}\right\}$.

To simplify notation, we will now assume that for all $n, H(n) \in \omega$. (If $H(n)$ are just arbitrary finite sets as in 2.1 , then we could prove analogous statements, replacing 0 and 1 by any two elements $0_{n} \neq 1_{n}$ of $H(n)$.)

2.25 Definition: Let $\underset{\sim}{f}$ be a $P T_{H}$-name for a function from $\omega$ to $\omega$. We say that $f$ splits on $p, k$ if for all $\eta \in \operatorname{split}_{k}(p)$ there are $l$ and $j_{1} \neq j_{0}$ such that

$$
\begin{aligned}
& p^{[\eta \frown 0]} \mid \vdash f(l)=j_{0} \\
& p^{[\eta-1]} \mid \vdash \underset{\sim}{\sim}(l)=j_{1}
\end{aligned}
$$

2.26 Remark: If $f$ splits on $p, k$, and $q \geq_{k+1} p$, then $f$ splits on $q, k$.

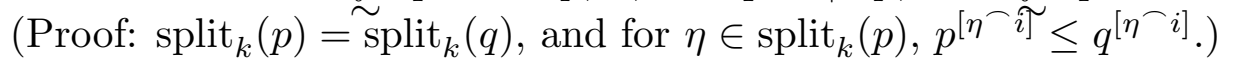

2.27 Lemma: If $p \mid \vdash f \notin V, k \in \omega$, then there is $q \geq_{k+1} p$ such that $f$ splits on $q, k$.

Proof: For $\eta \in \operatorname{split}_{k}(\widetilde{p}), i \in\{0,1\}$ we let $\eta_{i}$ be the unique element of $\operatorname{split}_{k+1}(p)$ satisfying $\eta \frown i \subseteq \eta_{i}$.

By 1.18, for each $\eta \in \operatorname{split}_{k}(p)$ we can find conditions $q_{\eta_{0}} \geq p^{\left[\eta_{0}\right]}, q_{\eta_{1}} \geq p^{\left[\eta_{1}\right]}$ and integers $l_{\eta}, j_{\eta, 0} \neq j_{\eta, 1}$ such that $q_{\eta_{0}} \vdash \vdash f\left(l_{\eta}\right)=j_{0}, q_{\eta_{1}} \vdash \vdash \underset{\sim}{f}\left(l_{\eta}\right)=j_{1}$. If $\nu \in \operatorname{split}_{k+1}(p)$ is not of the form $\eta_{0}$ or $\eta_{1}$ for any $\eta \in \operatorname{split}_{k}(p)$, then let $q_{\nu} \stackrel{\sim}{\sim}=p^{[\nu]}$.

By 2.11, $q:=\bigcup_{\nu \in \operatorname{split}_{k+1}(p)} q_{\nu}$ is a condition, $q \geq_{k+1} p$, and $q_{\nu}=q^{[\nu]}$ for all $\nu \in \operatorname{split}_{k+1}(p)$.

We finish the proof of 2.27 by showing that $f$ splits on $q, k$ : Let $\eta \in \operatorname{split}_{k}(p)=\operatorname{split}_{k}(q)$. Then $q^{\left[\eta^{-0} 0\right]}=q^{\left[\eta_{0}\right]}=q_{\eta_{0}}$, so $q^{\left[\eta^{-0}\right]} \mid \vdash f\left(l_{\eta}\right) \stackrel{\sim}{\sim} j_{\eta, 0}$. Similarly, $q^{\left[\eta^{-1}\right]} \mid \vdash f\left(l_{\eta}\right)=j_{\eta, 1}$.

2.28 Lemma: If $p \mid \vdash f \notin V$, then there is $q \geq p, \underset{\sim}{f}$ splits on $q, k$ for all $k$.

Proof: By 2.27, 2.26 and 2.9 (using a fusion argument).

2.29 Lemma: Assume $Q$ is a strongly $\omega_{\omega}$-bounding forcing notion. Let $f$ be a $Q$-name for a function, $p$ a condition, $n \in \omega, p \mid \vdash f \notin V$. Then there exists a natura numer $k$ such that

$$
\text { for all } \eta \in k^{k} \text { there is a condition } q \geq_{n} p, q \mid \vdash \underset{\sim}{\sim} \notin[\eta] \text {. }
$$

We will write $k_{p, n}$ or $k_{\sim, p, n}$ for the least such $k$. Note that for any $k \geq k_{p, n},(*)$ will also hold.

Proof: Assume that this is false. So for some $\underset{\sim}{f}, n_{0}, p_{0}$,

$$
\forall k \in \omega \exists \eta_{k} \in k^{k}: \neg\left(\exists q \geq_{n_{0}} p_{0} q \mid \vdash f \notin\left[\eta_{k}\right]\right)
$$


Let

$$
T:=\left\{\eta_{k} \mid l: l \leq k, k \in \omega\right\}
$$

$T$ is a finitely branching tree $\left(\subseteq \omega_{2}\right)$ of infinite height, so it must have an infinite branch. Let $f^{*} \in \omega_{2}$ be such that $\left\{f^{*} \mid j: j \in \omega\right\} \subseteq T$.

Since $f^{*} \in V$ but $p_{0} \vdash_{Q} \underset{\sim}{f} \notin V$, there exists a name $\underset{\sim}{m}$ of a natural number such that $p_{0}\left|\vdash f^{*}\right| \underset{\sim}{\sim} \neq \underset{\sim}{\sim} \mid \underset{\sim}{m}$. Let $q \underset{n_{0}}{\sim} p_{0}$ be such that for some $m^{*} \in \omega, q \mid \vdash \underset{\sim}{\sim}<m^{*}$.

Claim: For some $k, q \mid \vdash f \notin\left[\eta_{k}\right]$. This will contradict $(\star)$.

Proof of the claim: We have $q|\vdash f| m^{*} \neq f^{*} \mid m^{*}$. Since $f^{*} \mid m^{*} \in T$, there is a $k \geq m^{*}$ such that $f^{*}\left|m^{*}=\eta_{k}\right| m^{*}$. Hence $q|\vdash \tilde{\sim}| m^{*} \neq f^{*}\left|m^{*}=\eta_{k}\right| m^{*}$, so $q \mid \vdash f \notin\left[\eta_{k} \mid m^{*}\right]$. But then also $q \mid \vdash f \notin\left[\eta_{k}\right]$

This finishes the proof of the claim and hence of the lemma.

2.30 Lemma: Assume that $Q$ is a strongly $\omega_{\omega}$-bounding forcing notion, $\mathcal{H}$ is a dominating family in $V$, and $\bar{\nu}=\left\langle\nu^{h}: h \in \mathcal{H}\right\rangle$ has index $\mathcal{H}$. Then

$$
\vdash_{Q} \bigcap_{h \in \mathcal{H}} \bigcup_{k \in \omega}\left[\nu^{h}(k)\right] \subseteq V
$$

Proof: Assume that for some condition $p$ and some $Q$-name $\underset{\sim}{f}$,

$$
p \mid \vdash \underset{\sim}{f} \notin V \& \underset{\sim}{f} \in \bigcap_{h \in \mathcal{H}} \bigcup_{n \in \omega}\left[\nu^{h}(n)\right]
$$

We will define a tree of conditions such that along every branch we have a fusion sequence. Specifically, we will define an infinite sequence $\left\langle l_{n}: n \in \omega\right\rangle$ of natural numbers, and for each $n$ a finite sequence

$$
\left\langle p\left(\eta_{0}, \ldots, \eta_{n-1}\right): \eta_{0} \in \eta_{0} 2, \ldots, \eta_{n-1} \in \in^{l_{n-1}} 2\right\rangle
$$

of conditions satisfying

(0) $p()=p$

(1) For all $n: \forall \eta_{0} \in \eta_{02}, \ldots, \eta_{n-1} \in l^{l_{n-1} 2}: l_{n} \geq k_{p\left(\eta_{0}, \ldots, \eta_{n-1}\right), n}$.

(2) For all $n: \forall \eta_{0} \in \eta_{0} 2, \ldots, \eta_{n-1} \in{ }^{l_{n-1}} 2 \forall \eta_{n} \in{ }^{l_{n}} 2$

(a) $p\left(\eta_{0}, \ldots, \eta_{n-1}\right) \leq_{n} p\left(\eta_{0}, \ldots, \eta_{n-1}, \eta_{n}\right)$.

(b) $p\left(\eta_{0}, \ldots, \eta_{n-1}, \eta_{n}\right) \mid \vdash f \notin\left[\eta_{n}\right]$.

Given $p\left(\eta_{0}, \ldots, \eta_{n-1}\right)$ for all $\eta_{0} \in \eta_{0} 2, \ldots, \eta_{n-1} \in{ }^{l_{n-1}} 2$, we can find $l_{n}$ satisfying condition (1). The by the definition of $k_{p\left(\eta_{0}, \ldots, \eta_{n-1}\right), n}$ we can (for all $\left.\eta_{n} \in l_{n} 2\right)$ find $p\left(\eta_{0}, \ldots, \eta_{n-1}, \eta_{n}\right)$. Now let $h \in \mathcal{H}$ be a function such that for all $n, h(n)>l_{n}$. Define a sequence $\left\langle\eta_{n}: n \in \omega\right\rangle$ by $\eta_{n}:=\nu^{h}(n) \mid l_{n}$, and let $p_{n}:=p\left(\eta_{0}, \ldots, \eta_{n}\right)$. Then $p \leq p_{0} \leq_{0} p_{1} \leq_{1} \cdots$, so there exists a condition $q$ extending all $p_{n}$. So for all $n, q \mid \vdash f \notin\left[\eta_{n}\right]$. But then also for all $n$, $q \mid \vdash \underset{\sim}{f} \notin\left[\nu^{h}(n)\right]$, a contradiction. 
Lemma 2.30 will be needed later to show that if we iterate focings of the form $P T_{H}$ together with random real forcing, after $\omega_{2}$ many steps we obtain no strong measure zero sets of size $\aleph_{2}$. The proof (in 3.4) would be much easier if we could omit "strongly" from the hypothesis of 2.30, i.e., if we could answer the following question positively:

2.31 Open Problem: Assume $\mathcal{H} \subseteq \omega_{\omega}$ is a dominating family (or even wlog $\mathcal{H}=\omega_{\omega}$ ), and $\bar{\nu}$ has index $\mathcal{H}$. Let $Q$ be an $\omega_{\omega} \omega$-bounding forcing notion. Does this imply

$$
\vdash_{Q} \bigcap_{h \in \mathcal{H}} \bigcup_{n}\left[\nu^{h}(n)\right] \subseteq V ?
$$

2.32 Fact: Assume $h^{*}: \omega \rightarrow \omega-\{0\}, H^{*}(n)=h^{*}(n)$. Let $\mathcal{H} \subseteq \omega_{\omega}$ be a dominating family, and let $\bar{\nu}$ have index $\mathcal{H}$. Let $g^{*}$ be the name of the generic function added by $P T_{H^{*}}$.

Then

$$
\mid \vdash_{P T_{H^{*}}} \exists h \in \mathcal{H} \bigcup_{k \in \omega}\left[\nu^{h}(k)\right] \subseteq \bigcup_{n \in \omega}\left[g^{*}(n)\right]
$$

Proof: Assume not, then there is a condition $p$ such that

$$
p \mid \vdash \forall h \in \mathcal{H} \bigcup_{k \in \omega}\left[\nu^{h}(k)\right] \nsubseteq \bigcup_{n \in \omega}\left[g^{*}(n)\right]
$$

Let $h \in \mathcal{H}$ be a function such that $\forall k \in \omega \forall \eta \in \operatorname{split}_{2 k+1}(p) h^{*}(|\eta|) \leq h(k)$. This function $h$ will be a witness contradicting $(*)$.

For $\eta \in \operatorname{split}_{2 k+1}(p)$ let $i_{\eta} \in \operatorname{succ}_{p}(\eta)=H^{*}(|\eta|)=h^{*}(|\eta|) 2$ be defined by $i_{\eta}:=\nu^{h}(k) \mid h^{*}(|\eta|)$. (Note that $\nu^{h}(k) \in h(k)_{2}$ and $h(k) \geq h^{*}(|\eta|)$.)

Let $\vec{\imath}:=\left\langle i_{\eta}: \eta \in \operatorname{split}_{2 k+1}(p), k \in \omega\right\rangle$ and let $q:=p_{\vec{\imath}}$.

Then $q \mid \vdash \forall n \forall k\left(\underset{\sim}{g} \mid n \in \operatorname{split}_{2 k+1}(p) \Rightarrow \underset{\sim}{g}(n)=i_{g \mid n} \subseteq \nu^{h}(k)\right)$ by 2.24 .

Since also $q|\vdash \forall \widetilde{k} \exists n \underset{\sim}{g}| n \in \operatorname{split}_{2 k+1}(\widetilde{\widetilde{p}})$, we get $q \mid \vdash \forall k \exists n\left[\nu^{k}(k)\right] \subseteq[\underset{\sim}{g}(n)]$. This contra$\operatorname{dicts}(*)$.

\section{$\S 3$ Two models of $\operatorname{Add}(\mathcal{S})$.}

Recall that $S_{2}^{1}:=\left\{\delta<\omega_{2}: c f(\delta)=\omega_{1}\right\}$.

3.1 Lemma: Let $\left\langle P_{\alpha}, Q_{\alpha}: \alpha<\omega_{2}\right\rangle$ be an iteration of proper forcing noitions as in 1.8, $p \in P_{\omega_{2}}, \underset{\sim}{A}$ a $P_{\omega_{2}}$-name. If $p \mid \vdash \stackrel{\sim}{\sim}$ is a strong measure zero set," then there is a closed unbounded set $C \subseteq \omega_{2}$ and a sequence $\left\langle\bar{\nu}_{\delta}: \delta \in C \cap S_{2}^{1}\right\rangle$ such that each $\bar{\nu}_{\delta}$ is a $P_{\delta}$-name, and

$$
p \mid \vdash_{\omega_{2}} \bar{\nu}_{\delta} \text { has index } \omega_{\omega} \cap V_{\delta} \text { and } \underset{\sim}{A} \subseteq \bigcap_{h \in \omega_{\omega \cap V_{\delta}}} \bigcup_{n \in \omega}\left[\nu^{h}(n)\right]
$$

Proof: Let $\underset{\sim}{\sim}$ be a $P_{\omega_{2}}$-name for a function from $\omega_{2}$ to $\omega_{2}$ such that for all $\alpha<\omega_{2}$,

$$
\mid \vdash_{\omega_{2}} \forall h \in \omega_{\omega} \cap V_{\alpha} \exists \nu^{h} \in V_{\mathcal{\sim}(\alpha)}: \forall n \nu^{h}(n) \in{ }^{h(n)} 2 \& \underset{\sim}{A} \subseteq \bigcup_{n}\left[\nu^{h}(n)\right]
$$


(Why does $\underset{\sim}{\mathcal{c}}$ exist? Working in $V\left[G_{\omega_{2}}\right]$, note that there are only $\aleph_{1}$ many functions in $\omega_{\omega} \cap V_{\alpha}$, and for each such $h$ there is a $\nu^{h}$ as required in $\bigcup_{\beta<\omega_{2}} V_{\beta}$, by 1.7.)

As $P_{\omega_{2}}$ satisfies the $\aleph_{2}$-cc, by 1.6(1) we can find a function $c \in V$ such that $\mid \vdash_{\omega_{2}} \forall \alpha \underset{\sim}{c}(\alpha)<$ $c(\alpha)$. Let

$$
C:=\{\delta: \forall \alpha<\delta c(\alpha)<\delta\}
$$

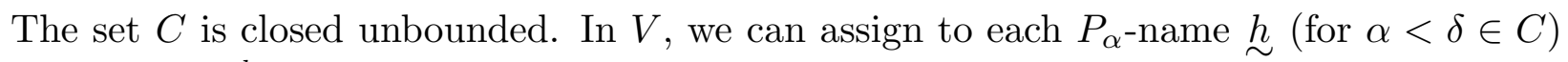
a $P_{\delta}$-name $\underset{\sim}{\sim} \stackrel{h}{\sim}$ such that

$$
\vdash_{\omega_{2}} \forall n \underset{\sim}{\sim} \sim \stackrel{h}{\sim}(n) \in \stackrel{h(n)}{\sim} 2 \& \underset{\sim}{A} \subseteq \bigcup_{n}\left[{\underset{\nu}{\sim}}_{\sim}^{h}(n)\right]
$$

Now in $V\left[G_{\delta}\right]$ we can choose for each $h \in \omega_{\omega}$ an $\alpha<\delta$ and a $P_{\alpha}$-name $\underset{\sim}{h}$ such that $h=\underset{\sim}{h}\left[G_{\delta}\right]$. Then we let $\nu^{h}:=(\underset{\sim}{\sim} \stackrel{h}{\sim})\left[G_{\delta}\right]$. Thus we found a sequence $\bar{\nu}=\left\langle\nu^{h}: \widetilde{h} \in V_{\delta}\right\rangle \in V_{\delta}$ as required.

\subsection{Lemma:}

Assume $\left\langle P_{\alpha}, Q_{\alpha}: \alpha<\omega_{2}\right\rangle$ is a countable support iteration of proper forcing notions, where for each ordinal $\delta \in S_{2}^{1} \mid \vdash_{\delta} Q_{\delta}=P T_{H_{\delta}}$ for some $P_{\delta}$-name $H_{\delta}$. We will write $g_{\delta}$ for the generic function added by $Q_{\delta}$.

Assume $\mathcal{H}$ is a name for a dominating family $\left(\subseteq{ }^{\omega}(\omega-\{0\})\right)$ in $V_{\omega_{2}}$, and

$$
\begin{array}{r}
\mid \vdash_{\omega_{2}} \text { "For all } h \in \underset{\mathcal{H}}{\sim}, S_{h}:=\left\{\delta<\omega_{2}: c f(\delta)=\omega_{1}, Q_{\delta}=P T_{H}{ }^{V_{\delta}}\right\} \\
\text { is stationary (where } H(n)={ }^{\left.h(n)_{2}\right) . "}
\end{array}
$$

Let $G_{\omega_{2}} \subseteq P_{\omega_{2}}$ be $V$-generic, then in $V\left[G_{\omega_{2}}\right]$, a set $A \subseteq \mathbb{R}$ is a strong measure zero set iff there is a closed unbounded set $C \subseteq \omega_{2}$ such that for every $\delta \in C \cap S_{2}^{1}, A \subseteq \bigcup_{n}\left[g_{\delta}(n)\right]$.

Proof: First we prove the easy direction. Assume that for some club $C$, for all $\delta \in C \cap S_{2}^{1}$, $A \subseteq \bigcup_{n}\left[g_{\delta}(n)\right]$. Then for every $h \in V_{\omega_{2}} \cap \omega(\omega-\{0\})$ there is a $\delta=\delta_{h} \in C \cap S_{h} \subseteq S_{2}^{1}$. So $Q_{\delta_{h}}=\left(P T_{H}\right)^{V_{\delta_{h}}}$, where $H(n)={ }^{h(n)_{2}}$. Since $g_{\delta_{h}}(n) \in{ }^{h(n)} 2$, and $A \subseteq \bigcup\left[g_{\delta_{h}}(n)\right]$ for arbitrary $h, A$ is a strong measure zero set.

Now for the reverse implication: In $V_{\omega_{2}}$, let $A$ be a strong measure zero set. By the previous lemma, there is a club set $C \subseteq \omega_{2}$ and a sequence $\left\langle\bar{\nu}_{\delta}: \delta \in C \cap S_{2}^{1}\right\rangle$ such that each $\bar{\nu} \in V_{\delta}$ is a sequence with index $\omega_{\omega} \cap V_{\delta}$ and $V_{\omega_{2}} \models A \subseteq X_{\bar{\nu}_{\delta}}$. By 2.32 we have for all $\delta \in C \cap S_{2}^{1}$ :

$$
V_{\delta+1} \models \exists h \in V_{\delta} \bigcup_{n}\left[\nu_{\delta}^{h}(n)\right] \subseteq \bigcup_{n}\left[g_{\delta}(n)\right]
$$

So fix $h_{0} \in V_{\delta}$ witnessing this. This inclusion is absolute, so also

$$
V_{\omega_{2}} \models \bigcup_{n}\left[\nu_{\delta}^{h_{0}}(n)\right] \subseteq \bigcup_{n}\left[g_{\delta}(n)\right]
$$


Thus

$$
V_{\omega_{2}} \models A \subseteq X_{\bar{\nu}_{\delta}} \subseteq \bigcup_{n}\left[\nu_{\delta}^{h_{0}}(n)\right] \subseteq \bigcup_{n}\left[g_{\delta}(n)\right]
$$

and we are done.

3.3 Corollary: Assume $P_{\omega_{2}}$ is as above. Then $\mid \vdash_{P_{\omega_{2}}} \operatorname{Add}(\mathcal{S})$.

Proof: Let $\left\langle A_{i}: i \in \omega_{1}\right\rangle$ be a family of strong measure zero sets in $V_{\omega_{2}}$. To each $i$ we can associate a closed unbounded set $C_{i}$ as in 3.2. Let $C:=\bigcap_{i \in \omega_{1}} C_{i}$, then also $C$ is closed unbounded, and for all $\delta \in C \cap S_{2}^{1}, \bigcup_{i \in \omega_{1}} A_{i} \subseteq \bigcup_{n \in \omega}\left[g_{\delta}(n)\right]$. Again by 3.2, $\bigcup_{i \in \omega_{1}} A_{i}$ is a strong measure zero set.

Our first goal is to show that $\operatorname{Unif}(\mathcal{S})$ does not guarantee the existence of a strong measure zero set of size $\mathbf{c}$. Clearly the model for this should satisfy $\mathbf{d}=\aleph_{1}$ (if $\mathbf{c}=\aleph_{2}$ ), so we will construct a countable support iteration of $\omega_{\omega}$-bounding forcing notions.

3.4 Theorem: If ZFC is consistent, then

$$
\begin{aligned}
\mathrm{ZFC}+\mathbf{c} & =\aleph_{2}+\mathcal{S}=[\mathbb{R}]^{\leq \aleph_{1}}+\text { no real is Cohen over } L \\
& + \text { there is a generalized Sierpinski set }
\end{aligned}
$$

is consistent.

Proof: We will start with a ground model $V_{0}$ satisfying $V=L$. Let $\mathcal{H}:={ }^{\omega}(\omega-\{0\}) \cap L=$ $\left\{h_{\alpha}: \alpha<\omega_{1}\right\}$, and let $H_{\alpha}(n)=h_{\alpha}(n) 2$.

Let $\left\langle S_{\alpha}: \alpha<\omega_{1}\right\rangle$ be a family of disjoint stationary sets $\subseteq\left\{\delta<\omega_{2}: c f(\delta)=\omega_{1}\right\}$.

Construct a countable support iteration $\left\langle P_{\alpha}, Q_{\alpha}: \alpha<\omega_{2}\right\rangle$ satisfying

(1) For all even $\alpha<\omega_{2}$ :

$$
\mid \vdash_{P_{\alpha}} \text { For some } h: \omega \rightarrow \omega-\{0\} \text {, letting } H(n)={ }^{h(n)} 2, Q_{\alpha}=P T_{H} .
$$

(2) If $\delta \in S_{\alpha}$, then $\mid \vdash_{\delta} Q_{\delta}=P T_{H_{\alpha}}$.

(3) For all odd $\alpha<\omega_{2}$ :

$$
\mid \vdash_{P_{\alpha}} Q_{\alpha}=\text { random real forcing. }
$$

By 1.11 (or as a consequence of 1.15), $P_{\omega_{2}}$ is ${ }^{\omega} \omega$-bounding, so $\mid \vdash_{\omega_{2}}$ " $\mathcal{H}$ is a dominating family." By 1.8(3) and 1.6 the assumptions of 3.3 are satisfied, so $\vdash_{\omega_{2}} \operatorname{Add}(\mathcal{S})$. Also, $\vdash \vdash_{\omega_{2}}$ "c $=\aleph_{2}$ and there are no Cohen reals over $L . "$ Letting $X$ be the set of random reals added at odd stages, $X$ is a generalized Sierpinski set: Any null set $H \in V_{\omega_{2}}$ is covered by some $G_{\delta}$ null set $H^{\prime}$ that coded in some intermediate model. As coboundedly many elements of $X$ are random over this model, $|H \cap X| \leq\left|H^{\prime} \cap X\right| \leq \aleph_{1}$.

To conclude the proof of 3.4 , we have to show

$$
V_{\omega_{2}} \models \text { "If } X \subseteq \mathbb{R} \text { is of strong measure zero, then }|X|<\mathbf{c} \text {." }
$$


Since $\mathcal{H}$ is a dominating family, by 0.4 it is enough to show that in $V_{\omega_{2}}$ the following holds:

If $\bar{\nu}$ has index $\mathcal{H}$, then $\left|X_{\bar{\nu}}\right| \leq \aleph_{1}$.

We will show: If $\bar{\nu} \in V_{\alpha}$ has index $\mathcal{H}$, then $X_{\bar{\nu}} \subseteq{ }^{\omega} \omega \cap V_{\alpha}$. (This is sufficient, by 1.7.) Assume to the contrary that $G_{\omega_{2}}$ is a generic filter, $\bar{\nu} \in V_{\alpha}$, and in $V\left[G_{\omega_{2}}\right]$ there is $\delta>\alpha$, $f \in V_{\delta}-\bigcup_{\gamma<\delta} V_{\gamma}, f \in X_{\bar{\nu}}$. So also

$$
V\left[G_{\delta}\right] \models f \in V_{\delta}-\bigcup_{\gamma<\delta} V_{\gamma} \text { and } f \in X_{\bar{\nu}}
$$

Let $\underset{\sim}{f}$ be a $P_{\delta}$-name, $\underset{\sim}{\bar{\nu}}$ a $P_{\alpha}$-name, and let $p \in P_{\delta}$ be a condition forcing all this. $\delta$ cannot be a successor ordinal, by 2.30. So $\delta$ is a limit ordinal, and $c f(\delta)$ must be $\omega$, otherwise we would have ${ }^{\omega_{2}} \cap V_{\delta}={ }^{\omega_{2}} \cap \bigcup_{\gamma<\delta} V_{\gamma}$.

So we have reduced the problem to the following lemma:

3.5 Lemma: Let $\left\langle P_{\alpha}, Q_{\alpha}: \alpha<\varepsilon\right\rangle$ be a countable support iteration of forcings where each $Q_{\alpha}$ (for even $\alpha$ ) is of the form $P T_{H_{\alpha}}$ for some $\left(P_{\alpha}\right.$-name) $H_{\alpha}$, and $Q_{\alpha}$ is random real forcing for odd $\alpha$. Let $\delta \leq \varepsilon$ be a limit ordinal of countable cofinality, and let $\underset{\sim}{f}$ be a $P_{\delta}$-name of a function in ${ }^{\omega} 2$ such that $\mid \vdash_{\delta} \forall \alpha<\delta \underset{\sim}{f} \notin V_{\alpha}$.

Let $\mathcal{H} \in V_{0}$ be a dominating family of functions, and assume that $\bar{\nu}$ has index $\mathcal{H}$. Then $\mid \vdash_{\delta} f \notin \bigcap_{h \in \mathcal{H}} \bigcup_{n \in \omega}\left[\nu^{h}(n)\right]$.

For notational simplicity, we again assume that for all even $\alpha, \mid \vdash_{\alpha}$ " $H_{\alpha}: \omega \rightarrow \omega$ (rather than $\left.H_{\alpha}: \omega \rightarrow 2^{<\omega}\right) . "$

Before we prove this lemma, we need the following two definitions (which make sense for any countable support iteration $\left.\left\langle P_{\alpha}, Q_{\alpha}: \alpha<\omega_{2}\right\rangle\right)$.

3.6 Definition and Fact: For $p \in P_{\varepsilon}, \alpha<\varepsilon, p|\alpha| \vdash p(\alpha) \leq \underset{\sim}{\sim} \in Q_{\alpha}$, we define $p \wedge \underset{\sim}{\sim}$ as follows: $(p \wedge \underset{\sim}{\sim})(\gamma)=p(\gamma)$ for $\gamma \neq \alpha$, and $(p \wedge \underset{\sim}{\sim})(\alpha)=\underset{\sim}{\sim}$.

Then $p \wedge \underset{\sim}{\sim} \in P_{\varepsilon}, p \wedge \underset{\sim}{\sim} \geq p$, and $(p \wedge \underset{\sim}{\sim})|\alpha=p| \alpha$, so in particular $p|\alpha| \vdash p \wedge \underset{\sim}{\sim} \in P_{\varepsilon} / G_{\alpha}$. Furthermore, $p|\alpha| \vdash(p \wedge \underset{\sim}{\sim})(\alpha)=\underset{\sim}{\sim}$.

Also, if $p(\alpha)=\underset{\sim}{\sim}$, then $p \wedge \underset{\sim}{\sim}=p$.

3.7 Definition and Fact: If $p \in P_{\alpha}, A$ a countable subset of $\varepsilon$, and $p \mid \vdash \underset{\sim}{\sim} \in P_{\varepsilon} / G_{\alpha} \& \underset{\sim}{\sim} \geq$ $p \& \operatorname{dom}(\underset{\sim}{r}) \subseteq \alpha \cup A$, then we define $p \wedge \underset{\sim}{\sim}$ as follows:

For $\gamma<\alpha,(p \wedge \underset{\sim}{\sim})(\gamma)=p(\gamma)$. For $\gamma \geq \alpha$ and $\gamma \in A,(p \wedge \underset{\sim}{\sim})(\gamma)=r(\gamma)$. Again, $p \wedge \underset{\sim}{\sim} \in P_{\varepsilon}, p \wedge \underset{\sim}{\sim} \geq p$, and $(p \wedge \underset{\sim}{\sim})|\alpha=p| \alpha$, so in particular $p|\alpha| \vdash p \wedge \underset{\sim}{\sim} \in P_{\varepsilon} / G_{\alpha}$. Also, if $p_{1} \leq p_{2}$, then $p_{1} \wedge \underset{\sim}{\sim} \leq p_{2} \wedge \underset{\sim}{\sim}$.

3.8 Proof of 3.5: $\operatorname{cf}(\delta)=\omega$, so we can find an increasing sequence $\left\langle\delta_{n}: n<\omega\right\rangle$ of even ordinals converging to $\delta$. Assume there is a condition $p$ forcing that $\underset{\sim}{f} \in \bigcap_{h \in \mathcal{H}} \bigcup_{n \in \omega}\left[\nu^{h}(n)\right]$. 
We will define sequences $\left\langle p_{n}: n<\omega\right\rangle$,

$$
\begin{aligned}
& \left\langle F_{n}: n<\omega\right\rangle, \\
& \left\langle\ell_{n}: n<\omega\right\rangle, \\
& \left\langle\underset{\underbrace{}_{n}}{\ell_{n}^{i}}: n \in \omega\right\rangle \\
& \left\langle p_{n}^{i}: n \in \omega, i \in\{0,1\}\right\rangle,
\end{aligned}
$$

such that the following hold: For each $n, p_{n}, p_{n}^{0}, p_{n}^{1}$ are conditions in $P_{\delta}, \delta_{n}$ is an even ordinal $<\delta, F_{n}$ is a finite subset of $\delta_{n}, \ell_{n}$ is an integer, and ${\underset{\sim}{n}}_{n}$ is a $P_{\delta_{n}}$-name for an element of $\omega^{<\omega}$. (We let $p_{0}=p, F_{0}=\emptyset, \ell_{0}=0, p_{0}^{1}=p_{0}^{0}=p_{0}, s_{0}=\emptyset$ ). For all $n>0$ we will have:

(1) $p_{n-1} \leq_{F_{n}, n} p_{n}$.

(2) $F_{n} \subseteq \delta_{n}, F_{n-1} \subseteq F_{n+1}, \bigcup_{k} \operatorname{dom}\left(p_{k}\right) \subseteq \bigcup_{k} F_{k}$.

(3) $\delta_{n-1} \in F_{n}$.

(4) $p_{n}\left|\delta_{n}\right| \vdash \underset{\sim}{s} n=\operatorname{stem}\left(p_{n}\left(\delta_{n}\right)\right)=\operatorname{stem}\left(p_{n-1}\left(\delta_{n}\right)\right)$

(5) For $i \in\{0,1\}, p_{n}^{i}=p_{n} \wedge p_{n}\left(\delta_{n}\right)^{\left[s_{n} i\right]}$.

(6) $p_{n}\left|\delta_{n}\right| \vdash_{\delta_{n}} " \exists l<\ell_{n} \exists j_{0} \neq j_{1} \forall i \in\{0,1\}: p_{n}^{i} \mid \vdash_{\delta_{n}, \delta} f(l)=j_{i}$."

Note that (5) implies:

(5') $p_{n}\left|\delta_{n}\right| \vdash p_{n}^{i} \in P_{\delta} / G_{\delta_{n}}$

and (6) implies

$\left(6^{\prime}\right)$ For all $\eta \in{ }^{\ell_{n} 2:} p_{n}\left|\delta_{n}\right| \vdash \exists i \in\{0,1\}: p_{n}^{i}\left|\vdash_{\delta_{n}, \delta} f\right| \ell_{n} \neq \eta$

[Proof of $(6) \Rightarrow\left(6^{\prime}\right)$ : In $V_{\delta_{n}}$, let $i \in\{0,1\}$ be such that $\eta(l) \neq j_{i}$, where $l$ is as in (6).]

Finally, let $q=\bigcup_{n} p_{n}$. Then $q\left|\delta_{n}\right| \vdash \operatorname{stem}\left(p_{n}\left(\delta_{n}\right)\right)=\operatorname{stem}\left(q\left(\delta_{n}\right)\right)={\underset{\sim}{s}}_{n}$ by (1), (3) and (4) and 2.7. Let $h^{*} \in \mathcal{H}$ be a function such that for all $n, \ell_{n}<h^{*}(n)$. So for all $n, \nu^{h^{*}}(n) \mid \ell_{n}$ is a well-defined member of $\ell_{n} 2$.

For each $n$, let $\underset{\sim}{\sim} n$ be a $P_{\delta_{n}}$-name of an element of $\{0,1\}$ such that

(6") $p_{n}\left|\delta_{n}\right| \vdash \stackrel{\sim}{n}^{n}|\vdash f| \ell_{n} \neq \nu^{h^{*}}(n) \mid \ell_{n}$

Now define a condition $q^{\prime}$ as follows: For $\alpha \notin\left\{\delta_{n}: n \in \omega\right\}, q^{\prime}(\alpha)=q(\alpha)$, and

$$
q^{\prime}\left(\delta_{n}\right)=q(\delta) \stackrel{\left[s_{n} \underset{\sim}{\sim} n\right]}{\sim}
$$

(This is a $P_{\delta_{n}}$-name.)

Claim: $q^{\prime} \geq q \geq p$ (this is clear) and $q^{\prime} \mid \vdash f \notin \bigcup_{n \in \omega}\left[\nu^{h^{*}}(n)\right]$.

To prove this claim, let $G_{\delta} \subseteq P_{\delta}$ be a generic filter containing $q^{\prime}$, and assume $f:=f\left[G_{\delta}\right]$ is in $\left[\nu^{h^{*}}(n)\right]$. Let $i_{n}:=\underset{\sim}{i}{ }_{n}\left[G_{\delta_{n}}\right]$. Now $q \in G_{\delta}$ implies $p_{n} \in G_{\delta}$, so in particular $p_{n} \mid \delta_{n}^{\sim} \in G_{\delta_{n}}$. Note that $\operatorname{stem}\left(q\left(\delta_{n}\right)\right)=\operatorname{stem}\left(p_{n}\left(\delta_{n}\right)\right)=s_{n}$, so $q^{\prime} \in G_{\delta} \& p_{n} \in G_{\delta}$ implies $p_{n}^{i_{n}} \in G_{\delta}$. Also, by (6") we have $q^{\prime} \mid \vdash f \notin \bigcup_{n}\left[s^{f^{*}}(n)\right]$, a contradiction.

This finishes the proof of 3.5 modulo the construction of the sequences $p_{n}, F_{n}$, etc.

First we fix enumerations $\operatorname{dom}(r)=\left\{\alpha_{r}^{m}: m \in \omega\right\}$ for all $r \in P_{\delta}$. We will write $\alpha_{n}^{m}$ for $\alpha_{p_{n}}^{m}$. 
Assume $p_{n-1}$ is given. Let $F_{n}:=\delta_{n} \cap\left(F_{n-1} \cup\left\{\alpha_{k}^{m}: k<n, m<n\right\} \cup\left\{\delta_{n-1}\right\}\right)$. This will take care of (2) and (3).

To define $p_{n}$, first work in $V\left[G_{\delta_{n}}\right]$, where $p_{n-1} \mid \delta_{n} \in G_{\delta_{n}}$.

We let $s_{n}:=\operatorname{stem}\left(p_{n-1}\left(\delta_{n}\right)\right)$.

We let $r_{0}:=p_{n-1} \wedge p_{n-1}\left(\delta_{n}\right)^{\left[s_{n}^{-} 0\right]}$, and $r_{1}:=p_{n-1} \wedge p_{n-1}\left(\delta_{n}\right)^{\left[s_{n}^{-1} 1\right]}$.

By 1.18, we can find $l, j_{0} \neq j_{1}$ and $r_{0}^{\prime}, r_{1}^{\prime}$ such that $r_{i}^{\prime} \geq r_{i}$, and $r_{i}^{\prime} \mid \vdash \underset{\sim}{f}(l)=j_{i}$.

We now define a condition $r \in P_{\delta} / G_{\delta_{n}}$ as follows:

$\cdot r\left|\delta_{n}=p_{n-1}\right| \delta_{n}$.

- $r\left(\delta_{n}\right)=r_{0}^{\prime}\left(\delta_{n}\right) \cup r_{1}^{\prime}\left(\delta_{n}\right) \cup \bigcup\left\{p_{n-1}\left(\delta_{n}\right)^{\left[s_{n} i\right]}: i \in \operatorname{succ}_{p_{n-1}\left(\delta_{n}\right)}\left(s_{n}\right)-\{0,1\}\right\}$.

$\left(\right.$ So $\operatorname{stem}\left(r\left(\delta_{n}\right)\right)=s_{n}$.)

- If $\gamma \in \operatorname{dom}\left(p_{n-1}\right) \cup \operatorname{dom}\left(r_{0}^{\prime}\right) \cup \operatorname{dom}\left(r_{1}^{\prime}\right)$ and $\gamma>\delta_{n}$, we let $r(\gamma)$ be a $P_{\gamma}$-name such that

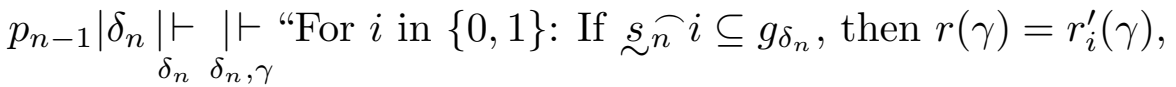

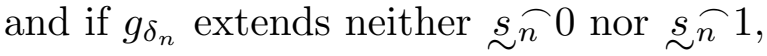

$$
\begin{aligned}
& \text { then } r(\gamma)=p_{n-1}(\gamma)
\end{aligned}
$$

(We write $g_{\delta_{n}}$ for $g_{Q_{\delta_{n}}}$, the branch added by the forcing $Q_{\delta_{n}}$.)

This is a condition in $P_{\delta} / G_{\delta_{n}}$. Note that we have the following:

(i) $\operatorname{stem}\left(p_{n}\left(\delta_{n}\right)\right)=s_{n}=\operatorname{stem}\left(p_{n-1}\left(\delta_{n}\right)\right)$

(ii) For $i \in\{0,1\}, r \wedge r_{i}^{\prime}\left(\delta_{n}\right) \geq r_{i}^{\prime}$.

(iii) $r \geq_{\delta_{n} \delta} p_{n-1}$.

Coming back to $V$, we can find names $\underset{\sim}{\sim}, \ldots$, such that the above is forced by $p_{n-1} \mid \delta_{n}$. Now let $\bar{r}$ be a condition in $P_{\delta_{n}}$ satisfying the following:

(a) $\bar{r} \geq_{F_{n}, n} p_{n-1} \mid \delta_{n}$.

(b) For some countable set $A \subseteq \delta, \bar{r} \mid \vdash \operatorname{dom}(\underset{\sim}{r}) \subseteq A$.

(c) For some $\ell_{n} \in \omega, \bar{r} \mid \vdash \underset{\sim}{l}<\ell_{n}$.

We can find a condition $\bar{r}$ satisfying (a)-(c) by 1.15 .

Finally, let $p_{n}:=\bar{r} \wedge \underset{\sim}{r}$. So $p_{n} \mid \delta_{n}=\bar{r}$.

And let $p_{n}^{i}$ be defined by (5).

Why does this work?

First we check (1): $p_{n-1}\left|\delta_{n} \leq_{F_{n}, n} p_{n}\right| \delta_{n}$ by (a), and $p_{n-1} \leq p_{n}$, because $p_{n}\left|\delta_{n}\right| \vdash p_{n}=$ $\bar{r} \wedge \underset{\sim}{\sim} \geq \underset{\sim}{\sim} \geq p_{n-1}$ (by (iii)). So $p_{n-1} \leq_{F_{n}, n} p_{n}$.

(2) and (3) are clear.

Proof of $(4): p_{n}\left|\delta_{n}\right| \vdash \operatorname{stem}\left(p_{n}\left(\delta_{n}\right)\right)=\operatorname{stem}\left((\bar{r} \wedge \underset{\sim}{r})\left(\delta_{n}\right)\right)=\operatorname{stem}\left(\underset{\sim}{r}\left(\delta_{n}\right)\right)=\underset{\sim}{s} n$.

(6): Let $G_{\delta_{n}}$ be a generic filter containing $p_{n} \mid \delta_{n}$. Work in $V\left[G_{\delta_{n}}\right]$. We write $r$ for $\underset{\sim}{r}\left[G_{\delta_{n}}\right]$, etc. 
We want to show $p_{n}^{0} \mid \vdash_{\delta_{n} \delta} f(l)=j_{0} \cdot\left(p_{n}^{1} \mid \vdash_{\delta_{n} \delta} f(l)=j_{1}\right.$ is similar. $)$ As $r_{0}^{\prime} \mid \vdash_{\delta_{n} \delta} f(l)=j_{0}$, it is enough to see $p_{n}^{0} \geq r_{0}^{\prime}$.

First we note that $p_{n}^{0} \geq p_{n} \geq p_{n-1}$. Also $p_{n}^{0}=p_{n} \wedge p_{n}\left(\delta_{n}\right)^{\left[s_{n} 0\right]} \geq p_{n}=\bar{r} \wedge r \geq r$.

Finally, $p_{n}^{0}\left(\delta_{n}\right)=r\left(\delta_{n}\right)^{\left[s_{n} 0\right]}=r_{0}^{\prime}\left(\delta_{n}\right)$.

So $p_{n}^{0}=p_{n}^{0} \wedge r_{0}^{\prime}\left(\delta_{n}\right) \geq r \wedge r_{0}^{\prime}\left(\delta_{n}\right) \geq r_{0}^{\prime}$, and we are done.

Our next model will satisfy

(*) $\quad \operatorname{Unif}(\mathcal{S})+\mathbf{d}=\mathbf{c}=\aleph_{2}$.

This in itself is very easy, as it is achieved by adding $\aleph_{2}$ Cohen reals to $L$. (Also Miller [10] showed that $\operatorname{Unif}(\mathcal{S})+\mathbf{c}=\aleph_{2}+\mathbf{b}=\aleph_{1}$ is consistent.)

Our result says that we can obtain a model for $(*)$ (and indeed, satisfying $\operatorname{Add}(\mathcal{S})$ ) without adding Cohen reals. In particular, $(*)$ does not imply $\operatorname{Cov}(\mathcal{M})$.

3.9 Theorem: Con(ZFC) implies

$$
\operatorname{Con}\left(\mathrm{ZFC}+\mathbf{c}=\mathbf{d}=\aleph_{2}>\mathbf{b}+\operatorname{Add}(\mathcal{S})+\text { no real is Cohen over } L\right)
$$

Proof (sketch): We will build our model by a countable support iteration of length $\omega_{2}$ where at each stage we either use a forcing of the form $P T_{H}$, or rational perfect set forcing. A standard bookkeeping argument ensures that the hypothesis of 3.3 is satisfied, so we get $\vdash{ }_{\omega_{2}} \operatorname{Add}(\mathcal{S})$. Using rational perfect set forcing on a cofinal set yields $\vdash_{\omega_{2}} \mathbf{d}=\mathbf{c}=\aleph_{2}$. Since all P-point ultrafilters from $V_{0}$ are preserved, no Cohen reals are added.

Proof (detailed version): Let $\left\{\delta<\omega_{2}: c f(\delta)=\omega_{1}\right\} \supseteq \bigcup_{\gamma<\omega_{2}} S_{\gamma}$, where $\left\langle S_{\gamma}: \gamma<\omega_{2}\right\rangle$ is a family of disjoint stationary sets. Let $\Gamma: \omega_{2} \times \omega_{1} \rightarrow \omega_{2}$ be a bijection. We may assume that $\delta \in S_{\Gamma(\alpha, \beta)} \Rightarrow \delta>\alpha$.

First we claim that there is a countable support iteration $\left\langle P_{\alpha}, Q_{\alpha}: \alpha<\omega_{2}\right\rangle$ and a sequence of names $\left\langle\left\langle\underset{\sim}{H_{\alpha}^{\beta}}: \alpha<\omega_{2}\right\rangle: \beta<\omega_{1}\right\rangle$ such that

(1) For all $\alpha<\omega_{2}$, all $\beta<\omega_{1},{\underset{\sim}{\sim}}_{\alpha}^{\beta}$ is a $P_{\alpha}$-name.

(2) For all $\alpha<\omega_{2}, \vdash_{\alpha}\left\{H_{\alpha}^{\beta}: \beta<\omega_{1}\right\}=\omega(\omega-\{0,1\})$.

(3) For all $\alpha<\omega_{2}$ : If $\alpha \notin \bigcup_{\gamma<\omega_{2}} S_{\gamma}$, then $\vdash_{\alpha} Q_{\alpha}=R P$.

(4) For all $\alpha<\omega_{2}$, all $\beta<\omega_{1}$, all $\delta \in S_{\Gamma(\alpha, \beta)}$ : $\mid \vdash_{\delta} Q_{\delta}=P T_{{\underset{\sim}{\alpha}}_{\alpha}^{\beta}}$.

Proof of the first claim: By induction on $\alpha$ we can first define $P_{\alpha}$, then $\left\langle\underset{\sim}{\sim}{ }_{\alpha}^{\beta}\right.$ : $\left.\beta<\omega_{1}\right\rangle$ (by $1.8(1)$ ), then $Q_{\alpha}$ (by (3) or (4), depending on whether $\alpha \in \cup_{\gamma<\omega_{2}} S_{\gamma}$ or not).

Our second claim is that letting $\underset{\mathcal{H}}{\sim}$ be a name for all functions from $\omega$ to $\omega-\{0,1\}$ in $V\left[G_{\omega_{2}}\right]$, the assumptions of 3.3 are satisfied, namely:

(a) $\mid \vdash_{\omega_{2}}$ " $\forall H \in \underset{\mathcal{H}}{\sim} \exists \gamma<\omega_{2} S_{\gamma} \subseteq S_{H}$."

(b) $\mid \vdash_{\omega_{2}}$ " $\forall \gamma<\omega_{2} S_{\gamma}$ is stationary."

(b) follows from 1.8(3) and 1.6, and (a) follows from

$\mid \vdash_{\omega_{2}}$ "For all $H \in \underset{\mathcal{H}}{\sim}$ there is $\alpha<\omega_{2}$ and $\beta<\omega_{1}$ such that $H=\underset{\sim}{H}{ }_{\alpha}^{\beta}$." 
which in turn is a consequence of 1.7.

So by $3.3, V_{\omega_{2}}=\operatorname{Add}(\mathcal{S})$.

Let $G_{\omega_{2}} \subseteq P_{\omega_{2}}$ be a generic filter, $V_{\omega_{2}}=V\left[G_{\omega_{2}}\right]$.

Again by 1.7 , every $\mathcal{H} \subseteq \omega_{\omega} \cap V_{\omega_{2}}$ of size $\leq \aleph_{1}$ is a subset of some $V_{\alpha}, \alpha<\omega_{2}$, so $\mathcal{H}$ cannot be a dominating family, as rational perfect set forcing $Q_{\alpha+1}$ will introduce a real not bounded by any function in $\mathcal{H} \subseteq V_{\alpha} \subseteq V_{\alpha+1}$. Hence $\mathbf{d}=\mathbf{c}=\aleph_{2}$.

Finally, any P-point ultrafilter from $V$ is generates an ultrafilter in $V_{\omega_{2}}$, so there are no Cohen reals over $V$.

This ends the proof of 3.9 .

\section{REFERENCES.}

[1] J. Baumgartner, Iterated forcing, in: Surveys in set theory (A. R. D. Mathias, editor), London Mathematical Society Lecture Note Series, No. 8, Cambridge University Press, Cambridge, 1983.

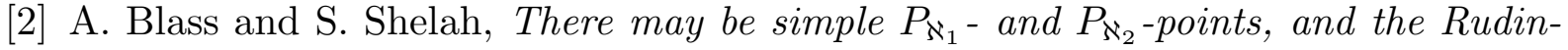
Keisler order may be downward directed, Annals of pure and applied logic, 33 (1987), pp.213-243.

[3] P. Corazza, The generalized Borel Conjecture and strongly proper orders, Transactions of the American Mathematical Society, 316 (1989), pp.115-140. .

[4] M. Goldstern, H. Judah, On Shelah's preservation theorem, preprint.

[5] H. Judah, S Shelah, H. Woodin, The Borel Conjecture, Annals of pure and applied logic, to appear.

[6] H. Judah, Strong measure zero sets and rapid filters, Journal of Symbolic logic, 53 (1988), pp.393-402.

[7] K. Kunen, Set Theory: An Introduction to Independence Proofs,

[8] A. Miller, Mapping a set of reals onto the reals, Journal of Symbolic logic, 48 (1983), pp. $575-584$.

[9] A. Miller, Rational Perfect Set Forcing, in: Axiomatic Set Theory, Boulder, Co 1983, 143-159, Contemporary Math 31, AMS, Providence RI 1984.

[10] A. Miller, Some properties of measure and category, Transactions of the American Mathematical Society, 266,1 (1981), pp.93-114.

[11] J. Pawlikowski, Power of transitive bases of measure and category, Proceedings of the American Mathematical Society, 93 (1985), pp.719-729. 
[12] Rothberger, Sur des families indenombrables de suites de nombres naturels et les problèmes concernant la proprieteé C, Proc. Cambr. Philos. Soc., 37 (1941), pp.109-126.

[13] Rothberger, Eine Verschärfung der Eigenschaft C, Fundamenta Mathematicae, 30 (1938), pp.50-55.

[14] S. Shelah, Proper Forcing, Lecture Notes in Mathematics Vol. 942, Springer Verlag.

[15] S. Shelah, Proper and Improper Forcing, to appear in Lecture Notes in Mathematics, Springer Verlag.

[16] R. Solovay and S. Tennenbaum, Iterated Cohen extensions and Souslin's problem, Annals of Mathematics, 94 (1971), pp.201-245.

[17] R. Solovay, Real valued measurable cardinals, in: Axiomatic Set Theory, Proc. Symp. Pure Math. 13 I (D. Scott, ed.), pp.397-428, AMS, Providence RI, 1971.

Martin GOLDSTERN goldstrn@bimacs.cs.biu.ac.il

Haim JUDAH judah@bimacs.cs.biu.ac.il

Department of Mathematics

Bar Ilan University

52900 Ramat Gan, Israel

Saharon SHELAH shelah@shum.huji.ac.il

Department of Mathematics

Givat Ram

Hebrew University of Jerusalem 